\title{
Developmental fidelity imposed by the RGL-1 "Balanced Switch" mediating opposing signals
}

Hanna Shin ${ }^{1 *}$, Christian Braendle ${ }^{3 *}$, Kimberly B. Monahan ${ }^{5 *}$, Rebecca E.W. Kaplan ${ }^{5 *}$, Tanya P. Zand $^{4,5}$, F. Sefakor Mote ${ }^{1}$, DeSean R. Craig ${ }^{5}$, Eldon Peters ${ }^{5}$, David J. Reiner ${ }^{1,2,4,5 \dagger}$

${ }^{1}$ Institute of Biosciences and Technology, Texas A\&M Health Science Center, Houston, TX, ${ }^{2}$ College of Medicine, Texas A\&M University, Houston, TX. ${ }^{3}$ Institut de Biologie Valrose, Universite Nice Sophia Antipolis, Parc Valrose, 06108 Nice cedex, France, ${ }^{4}$ Department of Pharmacology, ${ }^{5}$ Lineberger Comprehensive Cancer Center, University of North Carolina, Chapel Hill, NC 27599, USA,

${ }^{*}$ These authors contributed equally to this study

tTo whom correspondence should be addressed:

David J. Reiner

dreiner@ibt.tamhsc.edu 
Key Words: Ras, Raf, RalGEF, RalA, RaIB, PDK, Akt, PI3K, PTEN, cell fate, fidelity, robustness

\section{Abstract}

The six C. elegans vulval precursor cells (VPCs) are induced to form the $3^{\circ}-3^{\circ}-2^{\circ}-1^{\circ}-2^{\circ}-3^{\circ}$ pattern of cell fates with high fidelity. In response to EGF signal, the LET-60/Ras-LIN45/Raf-MEK-2/MEK-MPK-1/ERK canonical MAP kinase cascade is necessary to induce $1^{\circ}$ fate and synthesis of DSL ligands. In turn, LIN-12/Notch signal is necessary to induce neighboring cells to become $2^{\circ}$. We previously showed that, in response to lower dose of EGF signal, the modulatory LET-60/Ras-RGL-1/RalGEF-RAL-1/Ral signal promotes $2^{\circ}$ fate in support of LIN-12. In this study we identify two key differences between RGL-1 and RAL-1 functions. First, deletion of RGL-1 confers no overt developmental defects, while previous studies showed RAL-1 to be essential for viability and fertility. From this observation we hypothesize that the developmentally essential functions of RAL-1 are independent of upstream activation. Second, RGL-1 plays opposing and genetically separable roles in VPC fate patterning. RGL-1 promotes $2^{\circ}$ fate via canonical GEFdependent activation of RAL- 1 and $1^{\circ}$ fate via a non-canonical GEF-independent activity. Our genetic epistasis experiments are consistent with RGL-1 functioning in the modulatory $\quad 1^{\circ}$-promoting AGE-1/PI3-Kinase-PDK-1-AKT-1 cascade. Additionally, animals without RGL-1 experience 15-fold higher rates of VPC patterning errors compared to the wild type. Yet VPC patterning in RGL-1 deletion mutants is not more sensitive to environmental perturbations. We propose that RGL-1 functions as a 
"Balanced Switch" that orchestrates opposing $1^{\circ}$ - and $2^{\circ}$-promoting modulatory cascades to decrease inappropriate fate decisions. We speculate that such switches are broadly conserved but mostly masked by paralog redundancy or essential genes.

\section{Introduction}

Developmental patterning of the $C$. elegans vulva precursor cell (VPC) fates is a textbook system for analysis of cell-cell signaling. The vulva develops from six equipotent VPCs, P3.p through P8.p, which are induced to assume a $3^{\circ}-3^{\circ}-2^{\circ}-1^{\circ}-2^{\circ}-3^{\circ}$ pattern of cells fates. The anchor cell (AC) in the somatic gonad produces the LIN-3/EGF inductive signal (Fig. 1A). Historically, two models for VPC fate patterning have been advanced. The Morphogen Gradient Model posits that it is the position of each VPC in the LIN-3 gradient that dictates its fate. Notably, intact but isolated VPCs (the other five were killed by laser beam) could assume $1^{\circ}, 2^{\circ}$ or $3^{\circ}$ fates, depending on their distance from the AC. Modulation of EGF or EGFR dose could control these outcomes (1-4). Thus, these were consistent with a graded inductive signal.

In contrast, the Sequential Induction Model was posited upon identification and molecular genetic analysis of genes that are necessary and sufficient for the $1^{\circ}$ - and $2^{\circ}$ promoting VPC fate patterning signals. LIN-3 signals via the LET-23/EGFR-LET-60/RasLIN-45/Raf-MEK-2/MEK-MPK-1/ERK canonical MAP kinase cascade to induce $1^{\circ}$ fate (Fig. 1B; 5). LET-23 is necessary for $1^{\circ}$ but not $2^{\circ}$ fate $(6,7)$. However, these mosaic analyses did not address whether LET-23/EGFR might transduce a lower dose contribution to $2^{\circ}$ fate induction, consistent with the Morphogen Gradient Model.

The Sequential Induction Model was further supported by the discovery of redundant DSL ligands, produced by presumptive $1^{\circ}$ cells, that induce neighboring VPCs via LIN- 
$12 /$ Notch to become $2^{\circ}(8,9)$. LIN-12 is necessary and sufficient for $2^{\circ}$ fate $(10)$ and controls transcriptional client genes that were partially defined through computational means (Fig. 1B; 11-13).

Vulval induction is a stepwise progression: VPC fates are initially specified and later VPCs become committed (Fig. 1A; 14). Initial specification is accompanied by alterations of expression of certain signaling genes. Generally, expression of these is either uniformly absent or uniformly present in naive VPCs. Yet by the first cell division, several genes initiate lineage-specific expression patterns. Some of these transcriptional changes contribute to the Mutual Antagonism model, whereby contradictory signals are excluded from cells committing to cell fate. For example, the LIP-1/ERK phosphatase, a LIN-12 transcriptional client gene, is expressed in initially specified $2^{\circ} s$ to restrict inappropriate ERK activation $(11,12)$. Conversely, LIN-12, via mechanisms not entirely understood, is targeted for internalization and degradation in initially specified $1^{\circ}$ cells to restrict inappropriate $2^{\circ}$-promoting signal there (15-17).

Taken together, these many observations support the Sequential Induction model, yet fail to account for the experiments that implicate the action of an EGF gradient. The contradiction between these two models remained unresolved for 16 years. (18). We reconciled these potentially contradictory models by identifying the mechanism by which the $2^{\circ}$-promoting activity of the EGF gradient was transduced. We concluded that both models are true: initial sequential induction establishes the specification of the VPC pattern, and that overlaid on this pattern is a spatial EGF gradient that reinforces the initial pattern. To interpret different doses of EGF signal, LET-60/Ras dynamically switches effectors during VPC fate patterning, from LIN-45/Raf signaling through the canonical 
MEK-2/MEK-MPK-1ERK MAP kinase cascade to promote $1^{\circ}$ fate, to RGL-1/RalGEFRAL-1/Ral signaling to promote $2^{\circ}$ fate $(19,20)$.

We have further found that RAL-1 promotes $2^{\circ}$ fate through EXOC-8/Exo84, a subunit of the heterooctomeric exocyst complex, a known downstream oncogenic Ral signaling intermediary in mammalian cells. The GCK-2 MAP4 kinase and a downstream PMK1/p38 MAP kinase cascade is necessary and sufficient for Ral-dependent increased induction of $2^{\circ}$ cells, in support of the necessary and sufficient LIN-12 (Shin et al., in press).

More recently, this VPC fate patterning system has been exploited to investigate more sophisticated questions in developmental biology. Vulval development has been used to compare mechanisms of induction across nematode species both closely and distantly related to $C$. elegans (21-24). Additionally, VPC fate patterning has been used to assess heterogeneity in polymorphic wild C. elegans isolates $(22,25)$, and also developmental robustness in the face of environmental insult: the VPCs are pattered with $99.8 \%$ accuracy (26). But this accuracy is compromised by weak mutations in the signaling network that alter the delicate balance of $1^{\circ}$ and $2^{\circ}$ inductive signals (27-30). One potential extrapolation from these studies is that there are heretofore unidentified properties of signaling networks that increase fidelity and/or robustness.

Ras is the most mutated mammalian oncoprotein: more than a quarter of all tumors - up to $95 \%$ of Pancreatic Ductal Adenocarcinomas (PDAC) - harbor activating mutations in a Ras gene (31). Three main oncogenic Ras effector cascades have been identified (Fig. S1). Two of these, the Raf-MEK-ERK canonical MAP kinase cascade and the PI3Kinase-PDK-Akt cascade, are among the best-studied and most pharmacologically 
targeted cascades in all of biology (32-34). In contrast, the RalGEF-Ral effector signal is poorly characterized, though some argue it is at least as important for oncogenesis as Raf and PI3K cascades (35-40). Ras binds and activates RalGEF, an exchange factor that stimulates GTP loading on the Ral small GTPase. Ral is a Ras-like small GTPase subfamily, in the Ras family of the Ras superfamily, and is conserved throughout Metazoa (41). All Ras family members, including Ral, are similar structurally: they cycle between inactive GDP- and active GTP-bounds states, with the latter promoting effector binding. For most Ras family members, including mammalian RalA and RalB, this GDP/GTP cycle is controlled by activating GEFs and inactivating GAPs. And, despite their structural and primary sequence similarities, closely related GTPases in the same family diverge at the core effector binding sequence, in the Switch I region, that interfaces directly with effectors. Thus, Ral interacts with a very different set of effectors than does Ras (42).

Notably, the core effector-binding sequences of C. elegans LET-60 and RAL-1 are $100 \%$ identical to those of their Drosophila and mammalian counterparts $(20,41)$. Known orthologs of these effectors are also conserved across Metazoa, so the field has operated on the reasonable assumption that the different suites of effectors employed by Ras vs. Ral are conserved across species.

Mammals encode three Ras proteins (K-, N-, H-Ras), four GEFs with RA domains (RalGDS, RGL, RGL1, RGL2), two Rals (A, B), three Rafs (A-, B-, C-Raf) and three PI3Ks $(\alpha, \beta, \delta) . C$. elegans and Drosophila each harbor single Ral- and RalGEF-encoding genes, as well as single Ras-, Raf-, and PI3K-encoding genes (Fig. S1B). As is frequently the case with the multiplicity of mammalian genes compared to single invertebrate genes, 
these multiple isoforms likely impose an additional layer of complexity in signaling networks and cell biological outputs (43).

All three oncogenic Ras effectors have been implicated in C. elegans VPC fate patterning. Thus, we know of essential and modulatory cascades promoting both $1^{\circ}$ and $2^{\circ}$ fate: the essential LET-60-LIN-45 cascade promotes $1^{\circ}$ fate with support of the modulatory AGE-1-PDK-1-AKT-1 cascade, and the essential LIN-12/Notch cascade promoters $2^{\circ}$ fate with support of the modulatory LET-60-RGL-1-RAL-1 cascade.

Our analysis pursues the unexpected finding that RGL-1/RalGEF is functionally is non-equivalent to RAL-1 in two distinct ways. First, while RAL-1 is essential for viability and fertility $(20,44)$, RGL-1 is not essential. Second, RGL-1 and RAL-1 are nonequivalent in VPC fate patterning. While RAL-1 functions as a simple intermediary to propagate the $2^{\circ}$-promoting LET-60-RGL-1-RAL-1 signal, RGL-1 additionally performs an opposing function, a putative $1^{\circ}$-promoting function, that offsets its canonical $2^{\circ}$-promoting function. As a consequence, deletion of RGL-1 has no net effect on the delicate balance of $1^{\circ}$ - and $2^{\circ}$-promoting signals in our most sensitized mutant backgrounds. Using GEFspecific mutations and genetic bypass experiments, we show that the opposing functions of RGL-1 in VPC fate patterning are genetically separable and function cell autonomously in VPCs. In the context of mammalian studies that argue that RaIGEF physically interacts with PDK and Akt and functions as a scaffold $(45,46)$, our genetic epistasis results are consistent with RGL-1 functioning as a scaffold for PDK-1 and AKT-1 in the modulatory $1^{\circ}$-promoting AGE-1/PI3K cascade. Our analysis raises the question of how activity in two apparently opposing cascades contribute to VPC fate patterning. We found no difference in response of two RGL-1 deletion mutants and the wild type to environmental insults. Yet 
error rate in VPC patterning was 15-fold higher in the rgl-1 deletion mutants than in the wild type. We hypothesize that the two opposing activities of RGL-1, which tie together the two opposing $1^{\circ}$ and $2^{\circ}$-promoting modulatory cascades in VPC fate patterning, are orchestrated to reduce the level of noise in the signaling network, and hence reduce the rate of ambiguous fates or mis-patterning events. We conjecture that these properties of RGL-1 identify a novel switch to coordinate modulatory signaling activities in the reinforcement stage of VPC fate patterning that leads to fate commitment, and hence increases fidelity of the developmental process.

\section{Results}

The C. elegans RalGEF ortholog, RGL-1, is non-essential. We previously described that ral-1(tm2760) mutant animals are sterile but otherwise wild type. Efforts to feed or inject dsRNA in the RNAi hypersensitive rrf-3 background failed to phenocopy this sterility. Consistent with our depletion by bacterially mediated RNAi and injected dsRNA, tm2760 abrogated the $2^{\circ}$-promoting activity of RAL-1 (20).

Subsequent analysis of ral-1, including the more recently isolated allele, ral1(tm5205), led to the conclusion that RAL-1 function is maternally rescued and necessary for various facets of embryonic, post-embryonic, and germline development, including function of the PAR complex in cell polarity (44). This function is ascribed to a central role of RAL-1 in the exocyst complex, as described for mammals (47-52). Consistent with published results, we also observed that ral-1(tm5205) mutants become sickly in the fourth larval stage and become sterile adults, but that the VPCs are patterned normally $(N=52)$. 
Mammalian RalA and RalB associate with Sec5 and Exo84 subunits of the heterooctomeric exocyst complex (47-52). This functional association with the exocyst complex is consistent with results in C. elegans $(20,44)$, through the C-terminal membrane-targeting of Ral localizing the exocyst to specific subcellular domains to support certain exocyst functions. Yet mammalian studies suggest that Ral associates with the exocyst in an activity-dependent manner (47-52). This model predicts that abrogation of GTP-loading, either through blockade of total RalGEF activity or Ral GTPloading activity, should phenocopy loss of Ral and result in defective exocyst function. $C$. elegans, which encodes only a single RaIGEF ortholog, orthologous to mammalian RAdomain containing proteins (20), provides a system to test this relationship between RalGEF and Ral in vivo.

Also described previously, RNAi depletion of $r g l-1$ revealed a role in promoting $2^{\circ}$ fate (20). We reproduced RNAi depletion of rgl-1 with bacterially mediated and injected dsRNA in wild-type and rrf-3 mutant backgrounds and observed no overall development and fertility. Thus, we were unable to resolve this question by depletion of gene product via RNAi, and proceeded to analyze rgl-1 mutations not used in the previous study. Strikingly, four different deletion mutants of RGL-1 are superficially wild type (Table 1). By visual inspection, mutants lacking functional RGL-1 develop normally, are fertile, and can be grown indefinitely as homozygous mutant strains. One of these mutations, fax1(gm27), deletes several neighboring genes on the $X$ chromosome, including rgl-1 (53). Using robust primer sets (see Methods; Figs. S6, S7), we failed to amplify rgl-1 sequences from the gm27 mutant animal. 
We also previously published that nonsense mutants for the alpha or beta subunits of the heterodimeric RalGAP, HGAP-1 and HGAP-2, respectively, are also viable and fecund (54). The G26V putative activating mutation in RAL-1 also fails to confer developmental defects in an otherwise wild-type background (Shin et al., in press). The observation that deletion of RGL-1/RalGEF or HGAP-1/2/RaIGAP does not result in the same phenotype as deletion of RAL-1 raises the interesting possibility that Ral and RalGEF are functionally non-equivalent, contrary to the model derived from mammalian studies. Thus, our observations suggest that the role of RAL-1 in the exocyst is independent of GDP/GTP-bound state.

Sensitized genetic backgrounds reveal nuances in signaling. Four signaling cascades - two central and two modulatory - control $1^{\circ}$ and $2^{\circ}$ fate induction. We present a schematic of the signaling cascades discussed in this study (Fig. 1B,C; Fig. S1). Core $1^{\circ}$ - and $2^{\circ}$-promoting signals are detectable by direct mutation: since they are necessary and sufficient to induce their respective fates, mutational perturbation of them causes loss or gain of vulval cell types. In contrast, the role of the two modulatory cascades is not revealed through single mutant analysis, but rather requires sensitized genetic backgrounds. We propose that RGL-1/RaIGEF participates in both modulatory cascades (see below).

To detect such signals, we use let-60(n1046gf), a moderately activating G13E mutation analogous to mutations found in some mammalian cancers (55). In this background, gain and loss of the RAL- $12^{\circ}$-promoting signal resulted in decrease and increase of ectopic $1^{\circ}$ cells, respectively. We have similarly used the let-23(sa62gf) activating mutation in the LET-23/EGFR, and for under-induced backgrounds used the 
reduced function lin-45(n2506) background (20). Through combined use of genetic principles of parallelism and epistasis, we are able to dissect these modulatory signals. We present these principles as a network circuitry diagram (Fig. 1C). We have exploited such techniques to delineate a $2^{\circ}$-promoting signaling cascade downstream of RAL-1 (Shin et al., in press), and here use these techniques to similarly dissect the two roles of RGL-1/RalGEF in $2^{\circ}$ - and $1^{\circ}$-promoting activities.

\section{RGL-1 performs a function in VPC cell fate patterning that opposes its canonical}

$2^{\circ}$-promoting function. We previously showed that depletion of $r g l-1$ by RNAi revealed a role of RGL-1 in promoting $2^{\circ}$ fate consistent with the established Ras $>$ RalGEF>Ral signal in mammals (20). We reproduce these RNAi-based experiments here (Fig. 2A-C, E). Drawing from analysis of ral-1(tm5205) allele, which deletes RAL-1 sequences universally conserved in the small GTPase superfamily (44; our unpublished results), we analyzed the impact of tm5205 on VPC fate patterning. We found that ral-1(tm5205) confers enhancement of $1^{\circ}$ induction in the let-60(n1046gf) background (Fig. 2F).

To our surprise, analysis of various strong loss or putative null rgl-1 mutations in the let-60(n1046gf) background caused no net effect compared to the let-60(n1046gf) single mutant (Fig. 2D, F, G; see Table 1 and Fig 4A for rgl-1 alleles), contrasting with depletion of $r g l-1$ by injected or bacterially mediated RNAi. We replicated these results using a $1^{\circ}$ fate reporter that indicates neighboring $1^{\circ}$ cells in the let-60(gf) background $(12,20)$. Consistent with our previous results, rgl-1(RNAi) significantly increases the occurrence of neighboring $1^{\circ}$ cells, but rgl-1(tm2255) does not (Fig S2A-E). Conscious that the let60(n1046gf) mutant phenotype can drift and become more severe with protracted 
culturing, we used a stringent protocol for its analysis (20; Shin et al., in press; see Methods). To test that these results are not specific to the let-60(n1046gf) sensitized background or a $1^{\circ}$ over-inducing background, we also assessed the role of deleted rgl1 in under-induced background lin-45(n2506) and over-induced background let23(sa62gf) $(2,20) \cdot r g l-1(\operatorname{tm} 2255)$ did not alter the balance between $1^{\circ}$ and $2^{\circ}$ induction (Fig. S2F,G), consistent with our results in the let-60(n1046gf) background.

Our results are consistent with the working model that RGL-1 performs an additional, Ral-independent function that antagonizes its canonical function, perhaps by promoting $1^{\circ}$ fate. Consequently, deleting both functions results in no net change in the delicate balance between $1^{\circ}$-promoting and $2^{\circ}$-promoting signals in our sensitized genetic backgrounds. The discrepancy between RNAi- and mutational-based analyses is consistent with RGL-1 having different functional thresholds in level of gene product for the two opposing activities, which we have been unable to further investigate.

The rgl-1 transcriptional fusion is expressed in both $1^{\circ}$ and $2^{\circ}$ lineages. We previously described a transgenic ral-1 transcriptional fusion that expressed GFP dynamically over the time course of VPC fate induction (20). To summarize published results, early in the $3^{\text {rd }}$ larval stage (L3), GFP was expressed consistently in all six VPCs. Later in L3, after induction, GFP expression was excluded from presumptive $1^{\circ}$ cells while persisting in presumptive $2^{\circ}$ cells. These observations provided a potentially critical mechanistic insight: by reducing inferred RAL-1 expression in presumptive $1^{\circ}$ cells while retaining expression in presumptive $2^{\circ}$ cells, the signaling network could attenuate 
inappropriate RAL-1 activation in presumptive $1^{\circ}$ cells, thereby preventing potentially contradictory LET-60 signaling through RGL-1 -RAL-1 in presumptive $1^{\circ}$ cells.

We similarly tested the expression pattern of a transgene harboring the $\mathrm{rgl}-1$ promoter transcriptional fusion to GFP, sEx14985 (56). As with the ral-1 promoter transcriptional fusion, the transgenic rgl-1 reporter was expressed in all VPCs early in L3 (Fig. 3). But, unlike the ral-1 transcriptional fusion, the $r g /-1$ transcriptional fusion continued to express GFP in presumptive $1^{\circ}$ cells throughout vulval patterning, proliferation and morphogenesis. Persistent GFP expression from the $\mathrm{rgl}-1$ promoter is consistent with both canonical Ral-dependent and non-canonical Ral-independent functions of RGL-1, were the opposing Ral-independent function $1^{\circ}$-promoting rather than $2^{\circ}$-promoting .

Around the time of the first VPC cell division, GFP expression from the rgl-1 transcriptional fusion increases in presumptive $1^{\circ}$ cells relative to presumptive $2^{\circ}$ cells The significance of this change is unclear, since many transcriptional fusions of vulval signaling genes change expression patterns around this time $(11-13,20,57$; Rasmussen et al., re-submitted). Yet one interpretation is that increased expression of RGL-1 in presumptive $1^{\circ}$ cells accounts for resistance to rgl-1(RNAi) of this putative $1^{\circ}$-promoting activity of RGL-1.

\section{Tagged endogenous RGL-1 is expressed uniformly throughout VPC development.}

To validate the RGL-1 transcriptional fusion, we used the self-excising cassette (SEC) approach (58) to tag the endogenous 5' end of the endogenous rgl-1 locus with sequences encoding $m$ NeonGreen fluorescent protein (mNG, FP) and a 3xFlag epitope 
tag (Fig. S3). We observed mNG throughout vulval lineages, consistent with that observed with the rgl-1 promoter::gfp transcriptional fusion transgene.

\section{RGL-1 performs opposing GEF-dependent and GEF-independent functions in VPC}

fate patterning. A list of $r g l-1$ mutations and other genetic tools is shown (Fig. 4A, B; Table 1). The Million Mutation Project (MMP) described random sequence identification of a large collection of mutagenized $C$. elegans lines, demanding only that mutant animals be viable and fertile over many generations (59). We analyzed the sole non-synonymous mutation in ral-1, gk628801, which caused an R139H mutation. Arg-139 is conserved in all Ras family members in metazoans. Outcrossed ral-1(gk628801) single mutant vulvae were superficially wild type ( $\mathrm{N}=83$ ). In the let-60(n1046gf) background, ral-1(gk628801) increased $1^{\circ}$ induction (Fig. 4C), consistent with our previously published analysis using ral-1(RNAi) and deletion mutations (Fig. 2; 20). Thus, ral-1(gk628801) perturbs RAL-1 2promoting signaling but does not disrupt RAL-1 sufficiently to confer exocyst-associated phenotypes.

Of 30 total non-synonymous mutations in $r g l-1$, we considered two likely to perturb function. rgl-1(gk275304) causes an R361Q change. Arg-361 is conserved in all CDC25/RasGEF domains. rgl-1(gk275304) conferred significant increase in ectopic $1^{\circ}$ induction in the n1046gf background (Fig. 4D), consistent with disrupting GEF domain function and hence abrogating activation of RAL-1. rgl-1(gk275305) causes a W163* change, which did not alter ectopic $1^{\circ}$ induction in the let-60(n1046gf) background. Both outcrossed single mutant strains were superficially wild type ( $N=48$ and 61 , respectively). 
We therefore hypothesized that $r g /-1$ encodes GEF-dependent and GEF-independent activities.

Using the VPC-specific lin-31 promoter (60), in the let-60(n1046gf); rgl-1(tm2255) double mutant background we generated transgenic extrachromosomal arrays expressing VPC-specific rgl-1(+) or rgl-1(R324E), a mutation deficient in GEF catalytic activity ("GEF dead") in mammalian RalGDS (RalGEF; 61). Ectopic $1^{\circ}$ induction was scored in animals harboring extrachromosomal arrays, as indicated by pharyngeal GFP

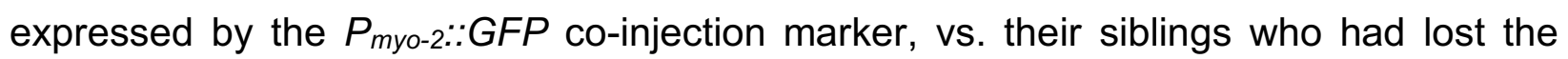
transgene. Animals bearing the R324E transgenes showed significant increase in ectopic $1^{\circ}$ induction. Animals bearing the wild-type transgenes were not different than their nonarray-bearing siblings (Fig. 4E). Consequently, we propose that RGL-1 performs GEFdependent and GEF-independent functions that are genetically separable by mutating the GEF domain. We also conclude that the GEF-independent RGL-1activity functions cell autonomously in the VPCs.

Transgenic animals expressing VPC-specific RGL-1(+) should restore responsiveness to ral-1(RNAi), while transgenic animals expressing putative GEF-dead VPC-specific RGL-1(R324E) should not. We evaluated responsiveness of the let60(n1046gf); rgl-1(tm2255) background harboring each transgene. reEx94/95 transgenic R324E animals failed to respond to ral-1(RNAi) compared to control gfp(RNAi) (Fig. S4A; the $1^{\circ}$ induction baseline is elevated due to rescue of the putative GEF-independent function shown in Fig. 4E). This observation was consistent with the absence of GEF activity. In contrast, animals harboring the reEx109/110/Plin-31::rgl-1(+)] transgene were responsive to ral-1(RNAi), resulting in increased $1^{\circ}$ induction, consistent with VPC- 
specific rescue of GEF activity and thus cell autonomy of the GEF-dependent function of RGL-1 (Fig. S4B).

Genetic bypass further reveals a GEF-independent activity of RGL-1. We previously showed that the ectopic vulva induction caused by mutation of lin-31, which confers ectopic $1^{\circ}$ induction, was insensitive to perturbation of LET-60-LIN-45-MEK-2-MPK-1 $1^{\circ}$ promoting signaling but sensitive to perturbation of LET-60-RGL-1-RAL-1 $2^{\circ}$-promoting signaling, consistent with the model of LIN-31 functioning downstream of ERK/MAPK-1. In that assay, rgl-1 function was assessed by RNAi, which resulted in increased $1^{\circ}$ induction, as did depletion of let-60 and ral-1 (and lin-12 positive control; 20). Here, rgl1(tm2255) similarly increased ectopic $1^{\circ}$ induction of lin-31(301) animals (Fig. S4C). This result is consistent genetically separable functions of RGL-1, and with the LIN-31/FoxB transcription factor functioning downstream of the putative non-canonical, GEFindependent signal, but in parallel to the canonical LET-60-RGL-1-RAL-1 signal (20).

We bypassed the requirement for GEF activity in RGL-1. We generated the rels10[Plin-

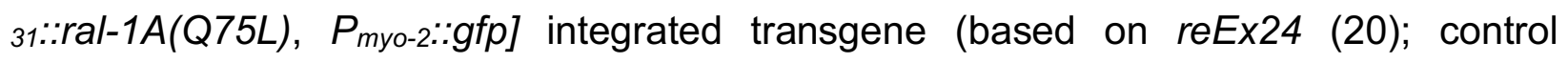
transgenes with RAL-1(+) had no effect). rels10 decreased ectopic $1^{\circ}$ induction in the let60(n1046gf) background (Fig. S4D). Into this background we additionally crossed the rgl1(tm2255) out-of-frame deletion mutation and scored the three strains concurrently. rgl1(tm2255) mutation significantly decreased ectopic $1^{\circ}$ induction below the level observed with rels10 alone, nearly to wild-type levels (Fig. 4F). This result is consistent with further deletion of a $1^{\circ}$-promoting activity being revealed when RGL-1 is deleted and the GEF activity is bypassed by activated RAL-1. Taken together, these experiments suggest that 
rgl-1 encodes two antagonistic signals, the GEF- and Ral-dependent $2^{\circ}$-promoting signal, and an unknown antagonistic signal, schematized in Fig. 4G.

RGL-1 may function in the $1^{\circ}$-promoting PI3K-PDK-Akt cascade. Mammalian RalGDS binds to PDK and Akt1 in cultured cells, possibly functioning as a scaffold for PDK and Akt $(45,46)$. The AGE-1/PI3K-PDK-1 signal has been described as promoting $1^{\circ}$ fate in VPC fate patterning (62), but AKT-1 was not implicated downstream of this process, potentially because of redundancy of AKT-1 and AKT-2 in C. elegans $(63,64)$. Previously, a gain-of-function mutation in AKT-1 was tested in a genetic background with reduced $1^{\circ}$ induction: no effect was found, leading to the model that AKT-1 did not contribute to vulval induction (62). We re-evaluated the akt-1(mg144gf) mutation in the let-60(n1046gf) background and observed significant increase in ectopic $1^{\circ}$ induction (Fig. $5 A)$. To corroborate previously published results, we assessed the impact of both activated AKT-1 and PDK-1 in the hypo-induced lin-45(n2506rf) background: akt1 (mg144gf) did not alter the hypo-induced lin-45(n2506rf) phenotype, but $p d k-1$ (mg142gf) did suppress the $1^{\circ}$-induction defect (Fig. S5C,D). This result, coupled with earlier analysis (62), suggests that the canonical AGE-1/PI3K-PDK-1/PDK-AKT-1/Akt cascade functions to promote $1^{\circ}$ vulval fate. We speculate that hypo-induced presumptive $1^{\circ}$ cells can respond to constitutively activated PDK-1 but not AKT-1, while in hyper-induced VPCs constitutively activated AKT-1 is sufficient to induce additional excess $1^{\circ}$ cells. Perhaps this distinction between PDK-1 and AKT-1 reflects the presence of AKT-2, for which we do not have activating mutations. 
Including the rgl-1(tm2255) mutation in the let-60(n1046gf); akt-1(mg144gf) background significantly suppressed ectopic $1^{\circ}$ induction, but not to the baseline of the n1046gf single mutant (Fig. 5A). Since we observed intermediate suppression, we constructed this strain twice and observed a similar result (Fig. S5A). We also reproduced this result with rgl-1(ok1921) and observed similar intermediate strength suppression that remained significantly above the baseline of the let-60(n1046gf) single mutant (Fig. S5B).

We further tested the relationship of RGL-1 with the rest of the PI3K cascade. Mutational activation of PDK-1 via the pdk-1(mg142gf) mutation also increased ectopic $1^{\circ}$ induction in the let-60(n1046gf) background. This effect was completely suppressed by rgl-1(tm2255) (Fig. 5B), suggesting a quantitatively detectable difference between the epistatic relationships of PDK-1 and AKT-1 with RGL-1. Genetic disruption of DAF18/PTEN, a negative regulator of this cascade in C. elegans in general $(65,66)$ and VPC fate patterning in particular (62), increases ectopic $1^{\circ}$ induction in the $n 1046 \mathrm{gf}$ background. We found that $r g l-1$ (tm2255) completely blocked this effect (Fig. 5B). The nonsense $r g l-1(g k 275305)$ but not the putative GEF dead $r g l-1(g k 275305)$ mutation similarly suppressed (Fig. S5E,F), suggesting that the putative RGL-1 $1^{\circ}$-promoting is GEF-independent.

While mammalian RalGDS bound directly to PDK, RalGDS bound indirectly to Akt through the intermediary scaffold, JIP (JNK Interacting Protein; $(45,46)$ ). Deletion the sole C. elegans JIP ortholog, JIP-1 (Fig. S5I) or RNAi depletion of JIP-1 (Fig. S5G) suppressed the ectopic $1^{\circ}$ phenotype of the daf-18(ok480) let-60(n1046gf) double mutant, consistent with JIP-1 collaborating with RGL-1 to scaffold PDK-1 and AKT-1 $1^{\circ}$-promoting signaling. RNAi depletion of both $p d k-1$ and $r g l-1$ similarly suppressed the enhanced $1^{\circ}$ 
induction of daf-18(ok480) let-60(n1046gf) (Fig. S5H). However, the jip-1 deletion allele enhanced n1046gf alone while suppressing daf-18(ok480) n1046. (Fig. S5I). We speculate that JIP-1 functions in the PI3K cascade, but may also function elsewhere in VPC fate patterning, perhaps in its canonical role as a scaffold for JKK and JNK MAP kinases. Further investigation of the role of JIP-1 is beyond the scope of this study.

A common target of the $C$. elegans PI3K-Akt cascade is inhibition of the DAF-16/FoxO transcription factor $(67,68)$. We tested the role of daf-16 alleles mu26, mu86 and mgDf47 in different backgrounds, with inconclusive results. Consequently, we were unable to determine the role, if any, of DAF-16/FoxO functions in VPC fate patterning.

Taken together, these genetic epistasis experiments, using an assay of parallelism with let-60(n1046gf), suggest that RGL-1 contributes to the AGE-1/PI3K-PDK-1/PDKAKT-1/Akt cascade, including the negative regulator lipid phosphatase, DAF-18/PTEN, in VPC fate patterning. Alone among the genetic tools used, the gain-of-function mutation in the downstream AKT-1 was only partially suppressed by rgl-1(tm2255), while the effect of other Akt cascade activators was completely suppressed by $\mathrm{rgl}-1$ (tm2255). We propose that RGL-1 is required for PDK-1 $1^{\circ}$-promoting signal (and AGE-/PI3K, through inference from our DAF-18/PTEN deletion experiments), but only partially required for AKT-1 $1^{\circ}$ promoting signal. We note that mammalian Akt is activated via parallel mechanisms: phosphorylation by upstream PDK and binding of $\mathrm{PIP}_{3}$, resulting in recruitment to the plasma membrane and activation (69). If RGL-1 functions as a scaffold for PDK-1 and AKT-1, its deletion would be expected to result in reduction of the PDK-1 phosphorylation of AKT-1 but not $\mathrm{PIP}_{3}$-dependent recruitment of AKT-1 to the plasma membrane. Thus, a parsimonious interpretation of our data is that RGL-1 functions as a 
scaffold for PDK-1-AKT-1 signaling in $1^{\circ}$ fate induction, and that this activity is independent of the GEF-dependent role of RGL-1 in promoting $2^{\circ}$ fate through $R A L-1$ activation. However, we cannot rule out the possibility of parallelism between a GEFindependent RGL-1 activity and the AKT-1 cascade, or RGL-1 functioning in a bifurcated cascade downstream of AKT-1 that is not revealed by the mechanism of constitutively activate PDK-1 or DAF-18/PTEN upstream.

Deletion of RGL-1 decreases fidelity of VPC patterning. Based on a relatively small sample size, RGL-1 deletions cause no gross VPC patterning defects, consistent with both cascades being modulatory, not central. We hypothesized that RGL-1 orchestrates activation of these two potentially opposing cascades - Akt output to presumptive $1^{\circ}$ cells and Ral output to presumptive $2^{\circ}$ cells - to improve robustness of the VPC developmental system in response to environmental stressors. A previous study investigated the impact on VPC fate patterning of environmental stressors in weakly disrupting genetic backgrounds, with an N2 baseline error rate of $0.2 \%$ under laboratory conditions (26). We similarly investigated the impact of environmental stressors of starvation, heat, and osmotic stress, compared to non-stressful conditions, on wild-type, rgl-1(ok1921), and rgl-1(tm2255) animals.

We evaluated 300 animals per genotype under each condition, totaling 1,200 animals per genotype and 3,600 animals overall (Fig 6A). To our surprise, the three measured environmental insults, compared to the unstressed, caused no patterning errors in either mutant or the wild type. However, the baseline patterning error rate was increased 15fold in rgl-1 mutants relative to the wild type, from $0.2 \%$ to $3.0 \%$, with no difference 
between the stressed and unstressed animals (Fig. 6A). These results suggest a generalized increase in error rate in the absence of functional RGL-1. Consequently, we propose that RGL-1 does not impact robustness in response to environmental stressors. Rather, we hypothesize that RGL-1 mitigates patterning error within the complex signaling system that regulates VPC patterning. This observation represents intriguing and unprecedented insight into the role of signaling networks in developmental fidelity.

\section{DISCUSSION}

The impetus for this analysis was two enigmatic genetic observations. First, deletion of RGL-1 confers no overt phenotype, in contrast to deletion of its signaling partner, RAL-1. Second, deletion of RGL-1 confers no net effect on $1^{\circ}$ vs. $2^{\circ}$ induction, while deletion or depletion of RAL-1 specifically reduces $2^{\circ}$ induction. Thus, in two different ways RGL-1 is non-equivalent to RAL-1. By pursuing these two genetic observations, we arrived at important models regarding the roles of RalGEF and Ral in signal transduction and development.

RAL-1 but not RAL-1 is essential for development. RAL-1 was previously implicated in developmental functions. Yet despite the ostensible linearity of Ras-RalGEF-Ral signaling, we unexpectedly found that RGL-1 is inessential for development. No role for LET-60/Ras has been found in exocyst and PAR complex function (70). Based on our previous analysis of the C. elegans RalGAP, neither do mutations in the HGAP-1/2 (54), predicted to confer excess activation of endogenous RAL-1. Nor does a constitutively active RAL-1 generated by CRISPR introduction of the G26V mutation into the 
endogenous ral-1 gene (Shin, et al., in press). Given that deletion of neither the GEF nor the GAP disrupts exocyst-dependent developmental events, but deletion of the small GTPase itself, RAL-1, does so, we hypothesize that the RAL-1 role as a membrane tether for the exocyst is independent of nucleotide-bound state. This hypothesis does not imply that there is no activation-dependent alteration of exocyst function by RAL-1, merely that such is not essential for the described developmental events.

An alternative hypothesis is that other GEFs or GAPs function redundantly with RGL1 and HGAP-1/2 to regulate the GDP/GTP cycle of RAL-1. Based on our analysis, RGL1 is required for GTP loading on RAL-1 for the $2^{\circ}$-promoting signal. But this other GEF would need to be specific for RAL-1 association with the exocyst and their functions in development, but not signaling to promote $2^{\circ}$ fate. While we cannot exclude this possibility, no GEFs fitting that role have been described in any system (RalGPS, a mammalian Ral-selective GEF that contains $\mathrm{SH} 3$ and $\mathrm{PH}$ domains, is not encoded in the C. elegans genome; (20), nor is TD-60/RCC2, similar to the Ran GEF RCC1 that controls nuclear import/export, which has been proposed to be an atypical GEF for RalA (71)).

\section{RGL-1 performs genetically separable and opposing functions in VPC fate} patterning. To our surprise, we found that deletion alleles of $\mathrm{rgl}-1$ caused no net alteration of the balance of $1^{\circ}$ and $2^{\circ} \mathrm{VPCs}$ in the let-60(gf) background. By genetic analysis, RGL1 performs its canonical role in promoting $2^{\circ}$ fate as a signaling intermediary between LET-60/Ras and RAL-1 ((20); this study), a role that mirrors extensive biochemical and cell biological evidence from mammalian cell culture (reviewed in $35,42,72$ ). Here, further genetic analysis reveals an additional, unexpected role for RGL-1, that of a non- 
canonical signaling participant opposing the canonical role as an activator of RAL-1. This non-canonical role apparently counteracts or "cancels out" the canonical role. In sensitized backgrounds, putative null mutations have no net effect on the sensitive balance between $1^{\circ}$ and $2^{\circ}$ VPC fates. We do not intend to imply that opposing functions are "equal," only that in most of our assays they appear to counter-balance each other with approximate equivalency. The observation that deletion of RGL-1 increase the basal error rate 15 -fold supports the idea that RGL-1 serves an important function, that of fidelity.

The key biochemical connection between RalGEF, PDK, and Akt came in mammalian cell culture, and in that context RalGEF-Ral and PDK-Akt cascades worked in concert (45). Yet historically RalGEF-Ral signaling has also been found to oppose canonical Ras effectors in cell culture (73). We hypothesize that the relationship between these cascades may depend on cell context, and in the case of vertebrates, which paralog of RalGEF is expressed.

RGL-1 is not the first protein in the VPC fate patterning network found to be bifunctional and promote both $1^{\circ}$ and $2^{\circ}$ fates. Depending on signaling dose and via mechanisms we do not understand, LIN-3/EGF-LET-23/EGFR signaling was found to promote both $1^{\circ}$ and $2^{\circ}$ fates, resulting in the original Morphogen Gradient Model $(1,2$, 4). We found that LET-60/Ras can promote $1^{\circ}$ or $2^{\circ}$ fate depending on its use of effector, LIN-45/Raf or RGL-1/RalGEF $(19,20)$. Yet the situations are not equivalent. LIN-3, LET23, and LET-60 are essential for vulval induction: strong loss results in complete absence of $1^{\circ}$ fate induction and hence a vulvaless phenotype (74-77), and thus their roles in $2^{\circ}$ fate induction were teased out only in sensitized backgrounds or special assays $(1,2$, 
20). In contrast, RGL-1 is dispensable for $1^{\circ}$ and $2^{\circ}$ fate induction, permitting dissection of its balanced and opposing functions. Furthermore, deletion of RGL-1 does not alter any other known developmental events.

These observations positioned us to discover an unexpected aspect of RGL-1 function: it contributes to two modulatory cascades, neither of which is essential for VPC induction. And since the two cascades promote opposing outcomes, loss of both together has no net effect on the delicate balance between $1^{\circ}$ and $2^{\circ}$ fates. Consequently, this unusual feature of RGL-1 function in vulval signaling, in the anatomically simple nematode that mostly lacks paralog redundancy, provided the opportunity to discover what may be a heretofore unknown property of signaling networks. We speculate that we have identified a signaling switch that is dispensable for development, and that precise regulation of this switch increases the fidelity of signaling networks and hence development. Specifically, in this case RGL-1 may orchestrate the opposing outputs of AKT-1, which promotes $1^{\circ}$ fate through unknown downstream targets (this study), and RAL-1, which promotes $2^{\circ}$ fate through EXOC-8/Exo84 of the exocyst, the GCK-2/MAP4 kinase, MLK-1/MAP3K, and PMK-1/p38 MAP kinase (Shin et al., in press).

\section{Linking opposed signaling cascades and mitigating development noise. The} problem that needs to be "solved" by the VPC fate patterning system is how to strictly define developmental fields in response to an initial point source of LIN-3/EGF ligand. In other words, how does one generate the precise $3^{\circ}-3^{\circ}-2^{\circ}-1^{\circ}-2^{\circ}-3^{\circ}$ pattern with $99.8 \%$ accuracy without mis-specified or ambiguous fates that might block mating and egg laying? A critical question, then, is how the programming of signal transduction networks 
decreases the potential for errors, or developmental "noise." Previously, the fidelity of VPC fate patterning was thought to be a property that emerges from the combination of three mechanisms: 1) Sequential Induction sets up the basic pattern, 2) the Morphogen Gradient collaborates with Sequential Induction to more precisely sculpt the initial pattern, and 3) Mutual Antagonism serves to exclude potentially conflicting signals from cells that are initially specified, thus preventing assumption of wrong or ambiguous fates. We speculate that the roles we describe here for RGL-1 define a fourth method that is woven into the other three: orchestration of two modulatory cascades to more sharply demarcate fates as a function of the VPC's spatial relationship to the AC and other VPCs. We name this property "Balanced Switches."

Yet is the participation of RGL-1 in two opposing cascades necessarily a novel mechanism that promotes fidelity? Perhaps not. Perhaps RGL-1 as a point of intersection between two conserved but opposing cascades is coincidental. In this alternative model, the loss of fidelity observed in deletion mutants of $r g l-1$ may be merely the consequence of losing two independent modulatory cascades, with AKT-1 and RAL-1 outputs, that each reinforces their respective fates. In this model, the loss of fidelity observed in rgl-1 deletion mutants is happenstance, a side effect of losing roughly equal and opposite modulatory cascades.

Alternatively, the wiring of these two cascades together in opposition could be of mechanistic significance. How such a mechanism would function is unclear. One possibility is that the RGL-1 "Balanced Switch" functions as an "Insulated Switch." The simplest way to envision this model is through mechanisms of subcellular recruitment and sequestration: RGL-1 engagement in one signal could physically exclude its engagement 
in the opposing signal, perhaps because the RGL-1 protein has been re-localized to a portion of the cell insufficient to participate in both signals, or is concomitantly modified to prevent interaction in its complementary function. For example, binding of Ras to the RA domain presumably recruits RGL-1 to the plasma membrane, consistent with Ras interactions with other effectors in mammalian cells. This recruitment could sequester RGL-1 away from the subcellular compartment in which PDK-1 and AKT-1 signal. Conversely, RGL-1 bound as a scaffold to PDK-1 and AKT-1 could be sequestered away from the subcellular compartment in which Ras interacts with RGL-1, the preventing participation in the canonical LET-60-RGL-1-RAL-1 $2^{\circ}$-promoting cascade. A metaphor for such a switch is the two-headed Pushmi-Pullyu from Dr. Doolittle: when one head pulls forward, the other is by necessity pulled back, and vice-versa. An inessential signaling protein with this property - like RGL-1 - could function to reinforce two different cell fates to improve developmental fidelity. And the mutually exclusive bi-directionality of such a switch would ensure that inappropriate signaling is minimized.

The mechanism by which RGL-1 contributes to different fates remains to be determined. We do not yet have biomarkers for RAL-1 or AKT-1 output in the VPCs. And because of the brief developmental window of VPC patterning and the tiny volume of VPC lineages relative to the entire animal, biochemical approaches are inadequate. The concept of the "Balanced Switch" being woven into signaling networks is a fascinating one, and one difficult to test with the typical manifold gene redundancy present in mammalian systems, or the essential nature of many signaling genes in the developmentally more complex Drosophila. Perhaps developmental patterning of the $C$. elegans vulva is the right place to test this idea, but more tools are needed to do so. Yet, 
in the dawn of the CRISPR era, we remain confident about our ability to do so in the future, including the possibility of generating two rgl-1 genes, each of which governs one by not the other function, i.e. uncoupling the two halves of the "switch."

Developmental fidelity vs. developmental robustness. Increasing noise could impact two obvious outcomes: fidelity and robustness. Fidelity here we define as developmental accuracy in the absence of external environmental perturbation, while robustness we define as developmental accuracy in the presence of perturbation. To our surprise, deletion of RGL-1 altered the fidelity but not robustness of VPC development: environmental perturbations had no effects on wild-type vs rgl-1 deletion mutant animals, while all mutant animals had a 15 -fold increase in patterning error rate compared to the wild type, regardless of their environment. Conversely, small changes in function, from weak mutation of certain cascades or introgression into different genetic backgrounds that might harbor mutations, caused increased sensitivity to environmental insult $(26,30)$. Yet, surprisingly, a four-fold change in EGF dose did not appreciably alter VPC patterning (27). We speculate that it is coordinate change caused by deletion of RGL-1 that does not decrease robustness. In other words, perhaps it is disruption of the delicate balance of $1^{\circ}$ - and $2^{\circ}$-promoting signals that sensitizes the system to environmental insult. Yet accumulation of mutations over many $(\sim 250)$ generations does increase error rate (28), and thus in these background mutations may be identified that disrupt fidelity-promoting functions like that of RGL-1. 
Conclusions. We define a putative regulatory system, which we term "Balanced Switching," that potentially mitigates potential signaling noise and hence developmental error. This system would likely not be discovered in experimental platforms with extensive paralog redundancy, or where the genes in question are essential for viability or the process being studied. Whether Balanced Switches are generalizable to other systems, and the molecular details by which they function, await further analysis.

In addition to divergent functions of RGL-1 in signaling, we also delineate mechanistic details of RAL-1 vs. RGL-1 function in essential functions performed by the exocyst and PAR complexes. Our results suggest that the role of RAL-1 in central machinery of cell biology is independent of GDP/GTP state, though we cannot rule out some switchable modulation of exocyst and PAR complex regulation by RAL-1. 
RGL-1/RalGEF in VPC fate patterning

Shin et al.

Table 1: rgl-1 and ral-1 alleles and mutant phenotypes

\begin{tabular}{|c|c|c|c|}
\hline Allele & Lesion & Superficial Phenotype & Net VPC alteration ${ }^{a}$ \\
\hline rgl-1(ok1921) & In-frame deletion, GEF domain & Wild type & none \\
\hline rgl-1(tm2255) & $\begin{array}{l}\text { Out-of-frame deletion, GEF } \\
\text { domain }\end{array}$ & Wild type & none \\
\hline fax-1(gm27) $)^{b}$ & rgl-1 and 8 other genes deleted & $U^{\prime n c^{c}}$ & none \\
\hline$r g l-1(g k 275305)$ & nonsense & Wild type & none \\
\hline rgl-1(gk275304) & Missense, R361Q, GEF domain & Wild type & Decreased $2^{\circ}$ \\
\hline ral-1(tm2760) & Intron 3 deletion & Sterile $^{d}$ & Decreased $2^{\circ} \mathrm{d}$ \\
\hline ral-1(tm5205) & Exons 2 and 3 deletion & Variable, Sterile $^{\mathrm{e}}$ & Decreased $2^{\circ} \mathrm{e}$ \\
\hline ral-1(gk628801) & Missense, $\mathrm{R} 139 \mathrm{H}$ & Wild type & Decreased $2^{\circ}$ \\
\hline ral-1(re179) & mNeonGreen::3xFlag::rgl-1 & Wild type & none $^{f}$ \\
\hline
\end{tabular}

a Alteration in network signaling inferred from phenotype in let-60(n1046gf) background ${ }^{\mathrm{b}}$ Documented in (53).

c Deletion of fax-1 causes an Uncoordinated phenotype (53).

${ }^{\mathrm{d}}$ Intron 2 deletion breaks in the middle of the splice donor site, conferring sterility and reduced $2^{\circ}$ induction (20).

${ }^{\mathrm{e}}$ Exons 2 and 3 deletion removes GTPase domain sequences, confers sterility, delayed growth and reduced $2^{\circ}$ induction (44), this study.

${ }^{f}$ No effect in let-60(n1046gf) background 
RGL-1/RalGEF in VPC fate patterning

Shin et al.

\section{Supplemental Information}

Supplemental information includes seven Supplemental figures.

\section{Methods}

C. elegans handling and genetics. Nomenclature is as described $(78,79)$. All strains were derived from the N2 wild type. Except where noted, animals were cultured on NGM agar plates with OP50 bacteria on at $20^{\circ} \mathrm{C}(80)$. Strains used are shown in Table S1. Data were analyzed with GraphPad Prism software (GraphPad Software Inc., La Jolla, CA).

PCR primers are listed in Table S2. For single animal genotyping PCR we used Taq PCR Master Mix (Qiagen): every reaction was run concurrently with +/+, m/+ and m/m control animals. The genotype of every PCR-genotyped strain was double-checked after completion of the strain construction. For each newly analyzed mutation, PCR products were sequenced to confirm break points. rgl-1(ok1921) was detected by triplex PCR using primers DJR614/615/616 (Tm: 59, 35 cycles), resulting in 366 bp (wild type) and 233 bp (ok1921) bands (Fig. S6). Point mutations in rgl-1 were tracked in trans to ok1921. Early constructions detected $r g l-1$ (tm2255) by triplex PCR using primers TZ20/DJR614/DJR615 (Tm: 58, 35 cycles), resulting in 913 bp (wild-type) and 595 bp (tm2255) bands (Fig. S7). Later constructions detected rgl-1(tm2255) by triplex PCR using primers FSM7/8/9 (Tm: 58 ${ }^{\circ}, 35$ cycles), resulting in 509 bp (wild-type) and 254 bp (tm2255) bands. pdk-1(mg142gf) was amplified by primers DRC1/2 (Tm: 60 35 cycles) to generate a $426 \mathrm{bp}$ band, and digested with Hpa II (NEB; 5 units added to total reaction volume doubled with water with NEB buffer \#1 to $0.5 x$ total, digested overnight at $37^{\circ} \mathrm{C}$ ). The band from the wild-type allele was digested to yield 126 and 300 bp bands, while the mg142 lesion abolishes the Hpa II site. daf-18(ok480) was detected by triplex PCR using primers FSM4/5/6 (Tm: 54 ${ }^{\circ}, 35$ cycles), resulting in 388 bp (wild-type) and 216 bp (ok480) bands. For this study, dpy-9(e14) was used as a balancer for daf-18(ok480), and the ok480 genotype confirmed after construction. akt-1(mg144gf) was 
balanced during strain constructions by dpy-11(e224) unc-76 (e905). ral-1(gk628801) was detected by amplification with primers DJR778/779 ( $\mathrm{Tm}=59.9,35$ cycles) to generate a $250 \mathrm{bp}$ band and digested with $\mathrm{HpyCH} 4$ IV (NEB; 5 units added to total reaction volume doubled with water with NEB buffer \#1 to $0.5 x$ total, digested overnight at $37^{\circ} \mathrm{C}$ ). The band from the wild-type allele was digested to yield 122 and 128 bp bands, while the gk628801 lesion abolishes the HypCH4 IV site.

Transgenic extrachromosomal array reEx24[Plin-31::ral-1(Q75L), $P_{\text {myo-2::gfp)] (20) was }}$ integrated by irradiation of late L4 animals using a Stratalinker (Stratagene) at dose of $12 \mathrm{~mJ} / \mathrm{cm}^{2}$.

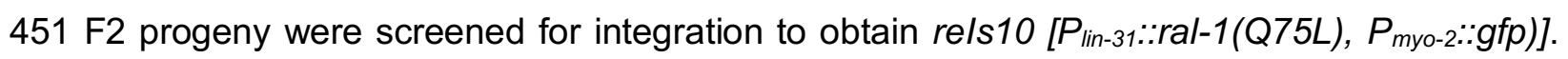
rels 10 was mapped to the region of Chromosome I +5 .

VPC induction assays. VPC induction was analyzed on an agar pad (molten $3 \%$ NG agar with $5 \mathrm{mM}$ sodium azide) on a slide. Late L4 animals were added to a $5 \mu \mathrm{l}$ drop of M9 buffer and the cover slip added. Ectopic pseudovulvae were scored at $600 x$ or $1000 x$ as invaginations using DIC/Nomarski optics (Nikon eclipse Ni) with images captured using NIS-Elements AR 4.20.00 software. Ectopic $1^{\circ}$ vulval induction index, from 0 to 3 ectopic $1^{\circ} \mathrm{s}$, is described elsewhere ((20); Shin et al in press). Under-induced backgrounds were scored as total VPCs induced, typically 03 (in under-induced backgrounds, $2^{\circ}$ lineages are occasionally missing). To summarize, we scored the wild-type vulval induction based on the stereotypical $2^{\circ}-1^{\circ}-2^{\circ}$ lineages centered on the $\mathrm{AC}$ at the A-P midpoint of the gonadal primordium (forming the "Christmas Tree" or "Stanley Cup" shape). In the let-60(n1046gf) and let-23(sa62gf) backgrounds, the morphology of ectopic $1^{\circ}$ cells generally conformed with the described "cap" structure where the entire $1^{\circ}$ lineage has pulled away from the cuticle (2). We did not observe ectopic pseudovulvae with the characteristic asymmetrical "beret" lineage of $2^{\circ}$ lineages, where one side remains attached to the cuticle.

As described previously ((20); Shin et al in press), we frequently observed drift of the severity of the let-60(n1046gf) but not let-23(sa62gf) Muv phenotype, generally increasing the severity of 
RGL-1/RalGEF in VPC fate patterning

Shin et al.

ectopic $1^{\circ}$ induction. For this study we analyzed parental MT2124 and outcrossed strains harboring the $n 1046 \mathrm{gf}$ single mutant, and established that undrifted strains average 1.2-1.5 ectopic $1^{\circ} \mathrm{s}$. (When grown on bacterially mediated RNAi food source HT115, induction was consistently higher; (20); Shin et al in press). For all strains harboring the $n 1046 \mathrm{gf}$ mutation we employed stringent scoring criteria: the $n 1046 \mathrm{gf}$ single mutant was scored first, and experiments deviating from the expected range of induction (1.2-1.5, 1.5-1.8 for RNAi) were discarded. We additionally always worked with freshly thawed or chunked strains, pulling from a large collection of undrafted strains that had been frozen. Thus, we always worked with animals that were freshly derived from a cross or from a thaw. When using this rigorous protocol, we rarely observe significant deviations from expected baselines. Additionally, we only compared genotypes that were scored concurrently, thus minimizing variation from assay to assay.

\section{Bacterially mediated RNA interference}

RNAi was performed as described previously ((20); Shin in press). RNAi plasmids used were: pREW2 (Iuciferase/luc; Shin et al. in press), X-2K11 (rgl-1), III-7M13 (ral-1), I-1K04 (pop-1), and gfp (20). Each RNAi clone was sequence verified. HT115 bacteria were used as the host for RNAi clones (81). Bacteria were grown on NGM plates supplemented with $50 \mu \mathrm{g} / \mathrm{ml}$ carbenicillin and 1 mM IPTG. Bacteria were grown (but not overgrown) overnight, without antibiotic selection. $80 \mu \mathrm{l}$ of fresh culture was seeded on plates on day 1, grown overnight, L4 animals were added on day 2 , transferred to a fresh plate on day 3 , and scored on day 5 .

Plasmid subcloning and transgene generation. Using primers RGL-1F and RGL-1R, the rgl1a cDNA was amplified from clone yk643d11, and digested with Bgl II and Not I. This isoform lacks exon 2, which by RNAseq data is rare (Wormbase WS263), yet still rescues, arguing that exon-2 is not required for vulval signaling. Plasmid vector pB255, which contains the lin-31 promoter and additional regulatory sequences and drives expression in VPCs (60), was digested 
RGL-1/RalGEF in VPC fate patterning

Shin et al.

with Bgl II and Not I to receive the rgl-1 insert. The putative R324E "GEF dead" mutation was introduced by PCR with Pfu Turbo using primers KM1 and KM2. The resulting plasmids, $\mathrm{P}_{\text {lin-31:: }}$ rgl-

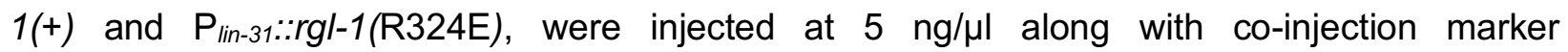

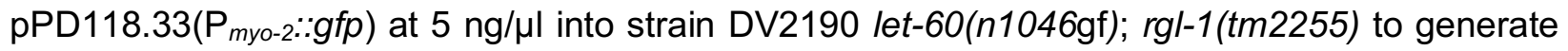
arrays reEx109 and reEx110 (rgl-1(+)) and reEx94 and reEx95 (rgl-1(R324E)), which express wild-type and “GEF dead” RGL-1, respectively, specifically in VPCs.

CRISPR/Cas9-dependent genome editing. rgl-1(re179[mNeonGreen::3xFlag::rgl-1]) was generated using the positive-negative selection self-excising cassette method (58). The repair template for rgl-1 5' tagging was generated by Gibson Assembly (NEB) with digested target SEC vector pDD268, and $\sim 500$ bp of homology arms amplified from genomic DNA by Q5 polymerase (NEB). We used two sgRNA sequences: (\#1) 5'-ACACCTTCGTATCCTTGTGGCGG-3' and (\#2) 5'-GGTCTGAGTTCTTCTGACGATGG-3', and hence generated two targeting vectors and one

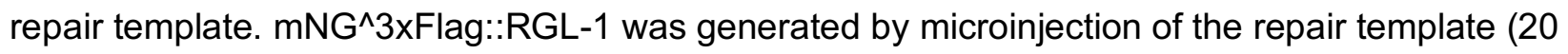

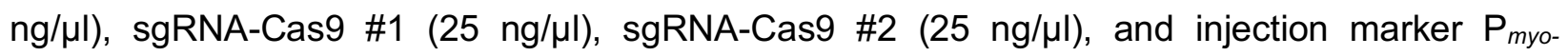
$2:: m$ Cherry $(2.5 \mathrm{ng} / \mathrm{\mu l})$ into wild-type animals. Knock-in alleles Genotyping and sequencing of $\mathrm{rg} /$ 15 ' CRISPR tagging was performed with HS125/126/127 (Tm=54). Validation was performed by western blotting using monoclonal anti-Flag antibody (Sigma-Aldrich F1804) (1:2000), monoclonal anti-a-tubulin antibody (Sigma-Aldrich T6199) (1:2000) and goat anti-mouse secondary antibody (MilliporeSigma 12-349) (1:5000).

For reasons unknown, all seven rgl-1 CRISPR alleles generated harbored mutations. Repair templates were re-checked by sequencing to confirm that sequences were wild type. DV3225 rgl1(re179[mNeonGreen::3xFlag::rgl-1]) harbored only promoter mutations (C insertion at -614, C deleted at $-375, C$ to $T$ at -395$)$ and so was selected for further analysis. Analysis of the ModEncode database showed no peaks of promoter occupancy at these sites. rgl1(re179[mNeonGreen::3xFlag::rgl-1]) had no effect on $1^{\circ}$ induction in the let-60(n1046gf) 
RGL-1/RalGEF in VPC fate patterning

Shin et al.

background $(P=0.57$ between strains with and without the re179 insertion, $N=86$ and 78 , respectively).

\section{DIC, epifluorescence and confocal microscopy.}

For DIC scoring of induction, live and appropriately staged animals were mounted in $5 \mathrm{mM}$ sodium azide/M9 buffer on slides with 3\% agar pad. For epifluorescent imaging, animals were mounted in $2 \mathrm{mg} / \mathrm{ml}$ tetramisole/M9 buffer and visualized using a Nikon Eclipse TE2000U microscope equipped with a DVC-1412 CCD camera (Digital Video Camera Company), with Hamamatsu SimplePCl acquisition software. Confocal images were captured by A1si Confocal Laser Microscope (Nikon) using NIS Elements Advanced Research, Version 4.40 software (Nikon).

\section{Assessment of patterning error rate with environmental insults.}

Large-scale vulval induction and environmental stressors were scored as previously described by Braendle et al. (26).

\section{Acknowledgements}

We thank Y. Kohara for the rgl-1 cDNA clone, the National Bioresource Project for the Experimental Animal Caenorhabditis elegans and the C. elegans Gene Knockout Consortium (Mitani, Barstead and Moerman labs) for deletion strains, D. Baillie for BC14985, and the Caenorhabditis Genetics Center (CGC), which is funded by NIH Office of Research Infrastructure Programs (P40 OD010440). We thank A. Fanning, B. Goldstein and K. Caron at UNC for microscope use and L. Vergara and the Center for Advanced Imaging at the TAMU Institute of Biosciences and Technology in Houston. We thank the Reiner lab for helpful discussions. This work was supported by NIH grant GM121625 to D.J.R.

\section{Figure Legends}


Figure 1: Schematics of VPC fate patterning and its signaling network. A) Initially equipotent VPCs are induced by the Anchor Cell (AC) to assume the $3^{\circ}-3^{\circ}-2^{\circ}-1^{\circ}-2^{\circ}-3^{\circ}$ pattern of fates (anterior-to-posterior), based on their position relative to the $A C$ (see Introduction). Over time, induced VPCs progress from naïve to initially specified to terminally committed to their fates, represented by equipotent and uninduced (gray) progressing through initially specified (hybrid colors with one color dominant) to terminally committed to $1^{\circ}$ (blue), $2^{\circ}$ (rose) or $3^{\circ}$ (yellow) fates. Yet the precise time course, molecular steps and network re-wiring events required for this progression are still unclear. B) The synthesis of the Sequential Induction, Morphogen Gradient, and Mutual Antagonism models of the VPC patterning signal transduction network, with the hypothesized RalGEF Balanced Switching mechanism superimposed. Names of human protein orthologs are used for ease of understanding between diverse experimental systems: EGF $=$ LIN-3, EGFR $=$ LET-23, Ras $=$ LET-60, Raf $=$ LIN-45, MEK $=$ MEK-2, ERK $=$ MPK-1, DSLs $=$ DSL ligands, Notch $=$ LIN-12, RalGEF $=$ RGL-1, Ral $=$ RAL-1, MKP $($ MAP kinase/ERK phosphatase $)=$ LIP-1, PTEN $=$ DAF-18, PI3K = AGE-1, PDK $=$ PDK-1, Akt $=$ AKT-1. Necessary and sufficient cascades are in dark colors (dark blue for $1^{\circ}$-promoting LET-23/EGFR-LET-60/Ras-LIN-45/Raf-MEK-2/MEK-MPK-1/ERK; dark rose for LIN-12/Notch-CSL transcriptional complex, CSL not pictured). Modulatory cascades are shown in lighter colors (light blue for $1^{\circ}$-promoting AGE-1/PI3K-PDK1/PDK-AKT-1/Akt, with light rose for inhibitory DAF-18/PTEN lipid phosphatase; light rose for $2^{\circ}$-promoting RGL-1/RalGEF-RAL-1/Ral). Green represents proteins capable of promoting $1^{\circ}$ or $2^{\circ}$ fate, like LIN-3/EGF, LET-23/EGFR and LET-60/Ras, depending on signal dose and as yet unknown factors. Mutual antagonism operates by excluding 
potentially contradictory signals from initially specified VPCs: in presumptive $1^{\circ}$ cells, LIN$12 /$ Notch is internalized and degraded (gray), while in presumptive $2^{\circ}$ cells MPK-1/ERK activation is repressed by transcriptional activation of LIP-1/MKP/DUSP/Erk phosphatase (and down-regulation of ral-1 promoter activity, not illustrated; (20)). The putative RGL1/RALGEF Balanced Switch is circled in purple both in presumptive $1^{\circ}$ and presumptive $2^{\circ}$ cells, and connected by a thin purple line to represent a hypothetical switch is its signaling activity. C) A wiring diagram of the naive $1^{\circ} / 2^{\circ} \mathrm{VPC}$ patterning signaling network, illustrating parallel and anti-parallel signals, with essential signals in dark blue and rose, and modulatory signals in light blue and rose. Data support RGL-1/RalGEF functioning in antagonistic $1^{\circ}$-promoting (non-canonical) and $2^{\circ}$-promoting (canonical) cascades. Deletion of $r g l-1$ would perturb both modulatory cascades but not alter the balance of $1^{\circ}$ and $2^{\circ}$-promoting signals, even in sensitized backgrounds.

Figure 2. RGL-1 and RAL-1 are functionally non-equivalent in VPC patterning. 1000x photomicrographs of late L4 A) wild-type, B) let-60(n1046gf); gfp(RNAi) vs. $600 \mathrm{x}$ photomicrographs of C) let-60(n1046gf); rgl-1(RNAi), and D) let-60(n1046gf); rgl$1\left(\right.$ tm2255) animals. White lines $=$ normal $2^{\circ}-1^{\circ}-2^{\circ}$ L4 vulvae, white arrows $=\mathrm{L} 4$ ectopic $1^{\circ}$ pseudovulvae. Black scale bars $=10 \mu \mathrm{m}$. Anterior is left and ventral down. E) RNAi depletion of rgl-1 and ral-1 enhance $1^{\circ}$ induction relative to $g f p(R N A i)$ control. Data are the mean \pm standard error of the mean (SEM). For statistical reasons single, non-pooled assays are shown, and white numbers represent animals scored therein. Significance was calculated by Kruskal-Wallis, Dunn test. Data shown were scored concurrently and are representative of 4 independent assays (this study) and 6 prior independent assays 
(20). (The let-60(n1046gf) $1^{\circ}$ induction baseline is consistently higher when grown on HT115 bacterially-mediated RNAi food compared to the standard OP50; (20); Shin et al. in press.) F) Deletion of ral-1 but not rgl-1 enhances ectopic $1^{\circ}$ induction by let60(n1046gf). Data shown are representative of 4 assays, each scored concurrently. G) Re-constructed strains show the same result: ok1921, $\operatorname{tm} 2255$ and $g m 27$ deletions fail to significantly enhance ectopic $1^{\circ}$ induction by let-60(n1046gf). Left: Three $n 1046-$ containing isolates, with and without rgl-1 mutations and scored concurrently. The concurrently scored MT2124 $1^{\circ}$ induction baseline was not significantly different from outcrossed lines DV2214 and DV2215 (see Table S1), N = 30 for each, and from assays that showed the most deviation of double mutants from the single mutant, but are still not significantly different. Right: DV2251 let-60(n1046gf); lon-2(e678) vs. DV2252 let60(n1046gf); lon-2(e678) gm27 animals scored concurrently but separate from the left group, representative of two assays. H) A general model for opposing RGL-1 GEF/RAL1-dependent and - independent functions (green $=$ bifunctional, blue $=1^{\circ}$-promoting, rose $=2^{\circ}$-promoting, see Fig. 1 )

\section{Figure 3: VPC expression pattern of the rgl-1 transcriptional GFP fusion over time.}

We used a combination of DIC analysis of VPCs and migration of the gonadal distal cells for staging to characterize the dynamic pattern of GFP expression from sEx14985 (56) over time. Initial expression in naïve VPCs is uniform. Around the time of induction, expression is restricted to presumptive vulval lineages. Later expression, after the first 
RGL-1/RalGEF in VPC fate patterning

Shin et al.

cell division, remains higher in $1^{\circ}$ than $2^{\circ}$ linages. Later stages show low levels of expression in surrounding non-vulval hypodermal cells.

Figure 4. RGL-1 encodes genetically separable functions. A) A schematic of the rgl1 gene and genetic reagents for this analysis. Green triangle $=$ mNeonGreen::3xFlag tag by CRISPR (Fig. S3). Light green exon 2: by RNAseq this is a rare mRNA species, and the 20 residues coded for by Exon 2 are not conserved among Drosophila and mammalian RalGEFs. Purple, pink and bright green lines: REM, GEF and RA (Ras Association) domains (The REM domain is a structural component of some but not all Ras GEFs). Red $g k$ alleles: W163* and R361Q mutations from the million mutation project. Orange: canonical R324E "GEF dead " mutation in transgenes reEx94 and reEx95. Light blue: coverage of bacterially mediated RNAi clone (library location; (82)). Red lines: sequences deleted by ok921, tm2255 and gm27 deletions (dotted line indicates that deletion ends out of frame, and thus introduces premature stop codons). B) An alignment of a portion of the RalGEF domain containing the canonical "GEF dead" R324E and gk275304 R361Q mutations (top to bottom: Human RGL2, in which the GEF dead mutation was validated, fly RalGEF, C. elegans RGL-1). C) The ral-1(gk628801) R139H missense mutation enhances $1^{\circ}$ induction in the let-60(n1046gf) background. Data shown were scored concurrently and are representative of two assays. D) rgl-1 mutation gk275304 (R361Q) but not $g k 275305\left(\mathrm{~W} 163^{*}\right)$ enhances $1^{\circ}$ induction in the let60 (n1046gf) background. Data shown were scored concurrently and are representative of two assays. E) Transgenic rescue of $\mathrm{rgl-1}(\mathrm{tm} 2255)$ in the let-60(n1046gf) background. Transgenic array-bearing animals harboring Plin-31::rgl-1 cDNA with Pmyo-2::gfp co- 
injection marker and their non-array-bearing siblings were scored. The reEx94 (shown) and reEx95 (not shown) R324E mutant transgenes enhanced $1^{\circ}$ induction relative to their non-transgenic siblings, as scored in separate concurrent assays for each array, suggesting that a GEF-independent $1^{\circ}$-promoting activity of RGL-1 functions cell autonomously in the VPCs. (reEx94 rgl-1(R324E) mutant transgenic animals failed to respond to ral-1(RNAi), Fig. S4C.) The reEx109 (shown) and reEx110 (not shown) wildtype transgenes failed to alter $1^{\circ}$ induction relative to their non-array-bearing siblings, as scored in separate concurrent assays for each array, suggesting that the GEF-dependent $2^{\circ}$-promoting activity of RGL-1 also functions cell autonomously in the VPCs. (reEx109 rgl-1(+) transgenic animals responded to ral-1(RNAi), Fig. S4D.) F) rels10[Plin-31::ral-

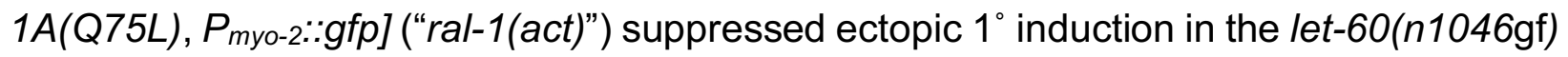
background; in a single concurrent assay, $1^{\circ}$ induction was further suppressed by rgl1(tm2255), revealing an opposing RGL-1 signal that may be $1^{\circ}$-promoting. Further validation of rels 10 "ral-1(act)" shown in Fig. S4B. Data are the mean \pm standard error of the mean (SEM). For statistical reasons single, non-pooled assays are shown, and white numbers represent animals scored therein. Significance was calculated by KruskalWallis, Dunn test. G) A schematic of the bypass experiment in F. Left: canonical and non-canonical RGL-1 activities are opposed and roughly equivalent (in the backgrounds assayed). Right: constitutive, VPC-specific activation of RAL-1 bypasses the GEF activity while also revealing a GEF-independent function of RGL-1, resulting in increased $2^{\circ}$ promoting signal and concomitant loss of $1^{\circ}$-promoting signal, with a net decrease of ectopic $1^{\circ}$ cells. 
Figure 5. RGL-1 interacts genetically with the $1^{\circ}$-promoting AGE-1/PI3K-PDK-1-AKT-1 cascade. A) The constitutively activating akt-1(mg144gf) mutation increased promotion of $1^{\circ}$ fate in the let-60(n1046gf) background, and this effect was partially blocked by rgl-1(tm2255), though the triple mutant was not suppressed to the double mutant baseline level. Animals were scored concurrently. These results were replicated with a re-built strain (Fig. S5A) and the strain with rgl1(ok1921) (Fig. S5B). Data are the mean \pm standard error of the mean (SEM). For statistical reasons single, non-pooled assays are shown, and white numbers represent animals scored therein. Significance was calculated by Kruskal-Wallis, Dunn test. B) The constitutively activating pdk-1(mg142gf) mutation increased promotion of $1^{\circ}$ fate in the let-60(n1046gf) background, and was completely suppressed to baseline level by rgl-1(tm2255). Animals were scored concurrently and are representative of two assays. C, D) Mutation of the negative regulatory PTEN ortholog by daf-18(ok480) similarly increased $1^{\circ}$ induction, and was completely suppressed to baseline level by rgl-1(tm2255) (C) and rgl-1(ok1921) (D). Animals for each were scored concurrently and scoring was repeated once, with the same general results. rgl-1(gk275305) (nonsense; Fig S5C) but not $r g l-1$ (gk265304) (GEF; Fig S5D) suppressed this ok480 enhancement, suggesting that the pertinent RGL-1 activity is GEF-independent/non-canonical.

Figure 6. Deletion of RGL-1 increases patterning errors but not susceptibility to environmental stress. A) rgl-1 deletions ok1921 and tm2255, introgressed to the wild type for eight generations (DV2696, DV2697), caused increased patterning defects compared to the wild type. ( $N=1,200$ per genotype, pooled from 300 for each condition.) Yet no difference was observed between animals subjected to stressors vs. control animals. $\mathrm{P}<0.0001$ for ok1921 or tm2255 animals vs. the wild type, calculated by Kruskal-Wallis, Dunn test. B) A model for the role of Balanced Switches in VPC fate patterning, in the context of Waddington's Developmental Topology. We hypothesize that through decreasing error rate, the RGL-1 signaling circuit effectively increases the "barrier" between $1^{\circ}$ and $2^{\circ}$ fates. 


\section{REFERENCES}

1. Katz WS, Hill RJ, Clandinin TR, \& Sternberg PW (1995) Different levels of the C. elegans growth factor LIN-3 promote distinct vulval precursor fates. Cell 82(2):297-307.

2. Katz WS, et al. (1996) A point mutation in the extracellular domain activates LET-23, the Caenorhabditis elegans epidermal growth factor receptor homolog. Mol Cell Biol 16(2):529-537.

3. Sternberg PW \& Horvitz HR (1986) Pattern formation during vulval development in C. elegans. Cell 44(5):761-772.

4. Sternberg PW \& Horvitz HR (1989) The combined action of two intercellular signaling pathways specifies three cell fates during vulval induction in C. elegans. Cell 58(4):679693.

5. Sundaram MV (2013) Canonical RTK-Ras-ERK signaling and related alternative pathways. WormBook:1-38.

6. Koga M \& Ohshima $Y$ (1995) Mosaic analysis of the let-23 gene function in vulval induction of Caenorhabditis elegans. Development 121(8):2655-2666.

7. Simske JS \& Kim SK (1995) Sequential signalling during Caenorhabditis elegans vulval induction. Nature 375(6527):142-146.

8. Chen N \& Greenwald I (2004) The lateral signal for LIN-12/Notch in C. elegans vulval development comprises redundant secreted and transmembrane DSL proteins. Dev Cell 6(2):183-192.

9. Zhang $X \&$ Greenwald I (2011) Spatial regulation of lag-2 transcription during vulval precursor cell fate patterning in Caenorhabditis elegans. Genetics 188(4):847-858.

10. Greenwald IS, Sternberg PW, \& Horvitz HR (1983) The lin-12 locus specifies cell fates in Caenorhabditis elegans. Cell 34(2):435-444.

11. Berset T, Hoier EF, Battu G, Canevascini S, \& Hajnal A (2001) Notch inhibition of RAS signaling through MAP kinase phosphatase LIP-1 during C. elegans vulval development. Science 291(5506):1055-1058.

12. Yoo AS, Bais C, \& Greenwald I (2004) Crosstalk between the EGFR and LIN-12/Notch pathways in C. elegans vulval development. Science 303(5658):663-666.

13. Yoo AS \& Greenwald I (2005) LIN-12/Notch activation leads to microRNA-mediated down-regulation of Vav in C. elegans. Science 310(5752):1330-1333.

14. Sternberg PW (2005) Vulval development. WormBook:1-28.

15. Deng Y \& Greenwald I (2016) Determinants in the LIN-12/Notch Intracellular Domain That Govern Its Activity and Stability During Caenorhabditis elegans Vulval Development. G3 (Bethesda).

16. Shaye DD \& Greenwald I (2002) Endocytosis-mediated downregulation of LIN-12/Notch upon Ras activation in Caenorhabditis elegans. Nature 420(6916):686-690.

17. Shaye DD \& Greenwald I (2005) LIN-12/Notch trafficking and regulation of DSL ligand activity during vulval induction in Caenorhabditis elegans. Development 132(22):50815092.

18. Kenyon C (1995) A perfect vulva every time: gradients and signaling cascades in C. elegans. Cell 82(2):171-174.

19. Reiner DJ (2011) Ras effector switching as a developmental strategy. Small GTPases 2(2):109-112.

20. Zand TP, Reiner DJ, \& Der CJ (2011) Ras effector switching promotes divergent cell fates in C. elegans vulval patterning. Dev Cell 20(1):84-96.

21. Felix MA \& Barkoulas M (2012) Robustness and flexibility in nematode vulva development. Trends Genet 28(4):185-195. 
RGL-1/RaIGEF in VPC fate patterning

Shin et al.

22. Grimbert S \& Braendle C (2014) Cryptic genetic variation uncovers evolution of environmentally sensitive parameters in Caenorhabditis vulval development. Evol Dev 16(5):278-291.

23. Sommer RJ \& Bumbarger DJ (2012) Nematode model systems in evolution and development. Wiley Interdiscip Rev Dev Biol 1(3):389-400.

24. Mahalak KK, Jama AM, Billups SJ, Dawes AT, \& Chamberlin HM (2017) Differing roles for sur-2/MED23 in C. elegans and C. briggsae vulval development. Dev Genes Evol 227(3):213-218.

25. Sterken MG, et al. (2017) Ras/MAPK Modifier Loci Revealed by eQTL in Caenorhabditis elegans. G3 (Bethesda) 7(9):3185-3193.

26. Braendle C \& Felix MA (2008) Plasticity and errors of a robust developmental system in different environments. Dev Cell 15(5):714-724.

27. Barkoulas M, van Zon JS, Milloz J, van Oudenaarden A, \& Felix MA (2013) Robustness and epistasis in the $C$. elegans vulval signaling network revealed by pathway dosage modulation. Dev Cell 24(1):64-75.

28. Braendle C, Baer CF, \& Felix MA (2010) Bias and evolution of the mutationally accessible phenotypic space in a developmental system. PLoS Genet 6(3):e1000877.

29. Duveau F \& Felix MA (2012) Role of pleiotropy in the evolution of a cryptic developmental variation in Caenorhabditis elegans. PLoS Biol 10(1):e1001230.

30. Milloz J, Duveau F, Nuez I, \& Felix MA (2008) Intraspecific evolution of the intercellular signaling network underlying a robust developmental system. Genes Dev 22(21):30643075.

31. Hobbs GA, Der CJ, \& Rossman KL (2016) RAS isoforms and mutations in cancer at a glance. Journal of cell science 129(7):1287-1292.

32. Fruman DA, et al. (2017) The PI3K Pathway in Human Disease. Cell 170(4):605-635.

33. Papke B \& Der CJ (2017) Drugging RAS: Know the enemy. Science 355(6330):11581163.

34. Wong KK, Engelman JA, \& Cantley LC (2010) Targeting the PI3K signaling pathway in cancer. Current opinion in genetics \& development 20(1):87-90.

35. Feig LA (2003) Ral-GTPases: approaching their 15 minutes of fame. Trends in cell biology 13(8):419-425.

36. Hamad NM, et al. (2002) Distinct requirements for Ras oncogenesis in human versus mouse cells. Genes Dev 16(16):2045-2057.

37. Lim KH, et al. (2005) Activation of RalA is critical for Ras-induced tumorigenesis of human cells. Cancer Cell 7(6):533-545.

38. Lim KH, et al. (2006) Divergent roles for RalA and RalB in malignant growth of human pancreatic carcinoma cells. Curr Biol 16(24):2385-2394.

39. Urano T, Emkey R, \& Feig LA (1996) Ral-GTPases mediate a distinct downstream signaling pathway from Ras that facilitates cellular transformation. The EMBO journal 15(4):810-816.

40. White MA, Vale T, Camonis JH, Schaefer E, \& Wigler MH (1996) A role for the Ral guanine nucleotide dissociation stimulator in mediating Ras-induced transformation. $J$ Biol Chem 271(28):16439-16442.

41. $\quad$ Reiner DJ \& Lundquist EA (2016) Small GTPases. WormBook:1-99.

42. Gentry LR, Martin TD, Reiner DJ, \& Der CJ (2014) Ral small GTPase signaling and oncogenesis: More than just 15minutes of fame. Biochimica et biophysica acta 1843(12):2976-2988.

43. Vigil D, et al. (2010) Aberrant overexpression of the Rgl2 Ral small GTPase-specific guanine nucleotide exchange factor promotes pancreatic cancer growth through Raldependent and Ral-independent mechanisms. J Biol Chem 285(45):34729-34740. 
RGL-1/RalGEF in VPC fate patterning

Shin et al.

44. Armenti ST, Chan E, \& Nance J (2014) Polarized exocyst-mediated vesicle fusion directs intracellular lumenogenesis within the C. elegans excretory cell. Dev Biol 394(1):110-121.

45. Hao Y, Wong R, \& Feig LA (2008) RalGDS couples growth factor signaling to Akt activation. Mol Cell Biol 28(9):2851-2859.

46. Tian X, Rusanescu G, Hou W, Schaffhausen B, \& Feig LA (2002) PDK1 mediates growth factor-induced Ral-GEF activation by a kinase-independent mechanism. The EMBO journal 21(6):1327-1338.

47. Chien Y, et al. (2006) RalB GTPase-mediated activation of the IkappaB family kinase TBK1 couples innate immune signaling to tumor cell survival. Cell 127(1):157-170.

48. Issaq SH, Lim KH, \& Counter CM (2010) Sec5 and Exo84 foster oncogenic rasmediated tumorigenesis. Molecular cancer research : MCR 8(2):223-231.

49. Jin R, et al. (2005) Exo84 and Sec5 are competitive regulatory Sec6/8 effectors to the RalA GTPase. The EMBO journal 24(12):2064-2074.

50. Moskalenko S, et al. (2002) The exocyst is a Ral effector complex. Nature cell biology 4(1):66-72.

51. Moskalenko S, et al. (2003) Ral GTPases regulate exocyst assembly through dual subunit interactions. J Biol Chem 278(51):51743-51748.

52. Sugihara K, et al. (2002) The exocyst complex binds the small GTPase RalA to mediate filopodia formation. Nature cell biology 4(1):73-78.

53. Much JW, Slade DJ, Klampert K, Garriga G, \& Wightman B (2000) The fax-1 nuclear hormone receptor regulates axon pathfinding and neurotransmitter expression. Development 127(4):703-712.

54. Martin TD, et al. (2014) Ral and Rheb GTPase activating proteins integrate mTOR and GTPase signaling in aging, autophagy, and tumor cell invasion. Molecular cell 53(2):209220.

55. Golden A (2017) From phenologs to silent suppressors: Identifying potential therapeutic targets for human disease. Mol Reprod Dev 84(11):1118-1132.

56. McKay SJ, et al. (2003) Gene expression profiling of cells, tissues, and developmental stages of the nematode C. elegans. Cold Spring Harb Symp Quant Biol 68:159-169.

57. Berset TA, Hoier EF, \& Hajnal A (2005) The C. elegans homolog of the mammalian tumor suppressor Dep-1/Scc1 inhibits EGFR signaling to regulate binary cell fate decisions. Genes Dev 19(11):1328-1340.

58. Dickinson DJ, Pani AM, Heppert JK, Higgins CD, \& Goldstein B (2015) Streamlined Genome Engineering with a Self-Excising Drug Selection Cassette. Genetics 200(4):1035-1049.

59. Thompson O, et al. (2013) The million mutation project: a new approach to genetics in Caenorhabditis elegans. Genome Res 23(10):1749-1762.

60. Tan PB, Lackner MR, \& Kim SK (1998) MAP kinase signaling specificity mediated by the LIN-1 Ets/LIN-31 WH transcription factor complex during $C$. elegans vulval induction. Cell 93(4):569-580.

61. Wolthuis RM, de Ruiter ND, Cool RH, \& Bos JL (1997) Stimulation of gene induction and cell growth by the Ras effector RIf. The EMBO journal 16(22):6748-6761.

62. Nakdimon I, Walser M, Frohli E, \& Hajnal A (2012) PTEN negatively regulates MAPK signaling during Caenorhabditis elegans vulval development. PLoS Genet 8(8):e1002881.

63. Paradis S, Ailion M, Toker A, Thomas JH, \& Ruvkun G (1999) A PDK1 homolog is necessary and sufficient to transduce AGE-1 PI3 kinase signals that regulate diapause in Caenorhabditis elegans. Genes Dev 13(11):1438-1452. 
RGL-1/RalGEF in VPC fate patterning

Shin et al.

64. Paradis S \& Ruvkun G (1998) Caenorhabditis elegans Akt/PKB transduces insulin receptor-like signals from AGE-1 PI3 kinase to the DAF-16 transcription factor. Genes Dev 12(16):2488-2498.

65. Gil EB, Malone Link E, Liu LX, Johnson CD, \& Lees JA (1999) Regulation of the insulinlike developmental pathway of Caenorhabditis elegans by a homolog of the PTEN tumor suppressor gene. Proc Natl Acad Sci U S A 96(6):2925-2930.

66. Ogg S \& Ruvkun G (1998) The C. elegans PTEN homolog, DAF-18, acts in the insulin receptor-like metabolic signaling pathway. Molecular cell 2(6):887-893.

67. Lin K, Dorman JB, Rodan A, \& Kenyon C (1997) daf-16: An HNF-3/forkhead family member that can function to double the life-span of Caenorhabditis elegans. Science 278(5341):1319-1322.

68. Ogg S, et al. (1997) The Fork head transcription factor DAF-16 transduces insulin-like metabolic and longevity signals in C. elegans. Nature 389(6654):994-999.

69. Vanhaesebroeck B \& Alessi DR (2000) The PI3K-PDK1 connection: more than just a road to PKB. Biochem J $346 \mathrm{Pt}$ 3:561-576.

70. Yochem J, Sundaram M, \& Han M (1997) Ras is required for a limited number of cell fates and not for general proliferation in Caenorhabditis elegans. Mol Cell Biol 17(5):2716-2722.

71. Papini D, et al. (2015) TD-60 links RalA GTPase function to the CPC in mitosis. Nat Commun 6:7678.

72. Kashatus DF (2013) Ral GTPases in tumorigenesis: emerging from the shadows. Exp Cell Res 319(15):2337-2342.

73. Goi T, Rusanescu G, Urano T, \& Feig LA (1999) Ral-specific guanine nucleotide exchange factor activity opposes other Ras effectors in PC12 cells by inhibiting neurite outgrowth. Mol Cell Biol 19(3):1731-1741.

74. Aroian RV, Koga M, Mendel JE, Ohshima Y, \& Sternberg PW (1990) The let-23 gene necessary for Caenorhabditis elegans vulval induction encodes a tyrosine kinase of the EGF receptor subfamily. Nature 348(6303):693-699.

75. Beitel GJ, Clark SG, \& Horvitz HR (1990) Caenorhabditis elegans ras gene let-60 acts as a switch in the pathway of vulval induction. Nature 348(6301):503-509.

76. Han M \& Sternberg PW (1990) let-60, a gene that specifies cell fates during C. elegans vulval induction, encodes a ras protein. Cell 63(5):921-931.

77. Hill RJ \& Sternberg PW (1992) The gene lin-3 encodes an inductive signal for vulval development in C. elegans. Nature 358(6386):470-476.

78. Horvitz HR, Brenner S, Hodgkin J, \& Herman RK (1979) A uniform genetic nomenclature for the nematode Caenorhabditis elegans. Molecular \& general genetics : MGG 175(2):129-133.

79. Dickinson DJ, Ward JD, Reiner DJ, \& Goldstein B (2013) Engineering the Caenorhabditis elegans genome using Cas9-triggered homologous recombination. Nature methods 10(10):1028-1034.

80. Brenner S (1974) The genetics of Caenorhabditis elegans. Genetics 77(1):71-94.

81. Timmons L \& Fire A (1998) Specific interference by ingested dsRNA. Nature 395(6705):854.

82. Kamath RS, Martinez-Campos M, Zipperlen P, Fraser AG, \& Ahringer J (2001) Effectiveness of specific RNA-mediated interference through ingested double-stranded RNA in Caenorhabditis elegans. Genome biology 2(1):Research0002. 


\section{Supplementary Table 1 - Strains}

\begin{tabular}{|c|c|c|}
\hline Strain & Genotype & Application \\
\hline DV3311 & $\begin{array}{l}\text { ral-1(tm2760) / qC1 [dpy-19(e1259ts) glp-1(q339) } \\
\text { nls189[P myo-2::gfp)]] }\end{array}$ & $\begin{array}{l}\text { Inspecting } \\
\text { mutant VPCs }\end{array}$ \\
\hline DV2924 & $\begin{array}{l}\text { ral-1(tm5205) / qC1 [dpy-19(e1259ts) glp-1(q339) } \\
\text { nls189[P } P_{\text {myo-2::gfp]] }}\end{array}$ & $\begin{array}{l}\text { Inspecting } \\
\text { mutant VPCs }\end{array}$ \\
\hline MT2124 & let-60(n1046gf) IV & $\begin{array}{l}\text { Fig. } 2 \mathrm{~B}, \mathrm{C}, \mathrm{E}, \\
\text { baselines } \\
\text { throughout }\end{array}$ \\
\hline DV2214 & let-60(n1046gf) IV ( $2 x$ outcrossed) & Results \\
\hline DV2215 & let-60(n1046gf) IV ( $2 x$ outcrossed) & Results \\
\hline RB1576 & rgl-1(ok1921) X & Starting reagent \\
\hline DV2194 & rgl-1(ok1921) X (4x outcrossed) & throughout \\
\hline $\mathrm{F} \times 2255$ & rgl-1(tm2255)X & Starting reagent \\
\hline DV2175 & rgl-1 $(\operatorname{tm} 2255) \times(4 \times$ outcrossed $)$ & throughout \\
\hline DV2937 & 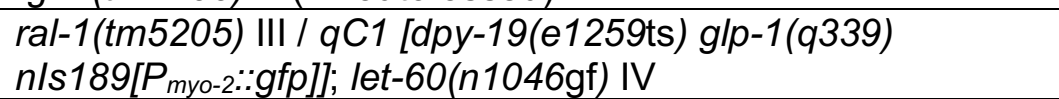 & Fig. $2 \mathrm{~F}$ \\
\hline DV2190 & let-60(n1046gf) IV; rgl-1(tm2255) X & Fig. 2D,F,G \\
\hline DV2248 & let-60(n1046gf) IV; rgl-1(ok1921)X & Fig. 2F,G \\
\hline DV2251 & let-60(n1046gf) IV; Ion-2(e678) X & Fig. 2G \\
\hline DV2252 & let-60(n1046gf) IV; fax-1(gm27) lon-2(e678) X & Fig. $2 \mathrm{G}$ \\
\hline DV2191 & $\begin{array}{l}\text { let-60(1046gf) IV; arls92[Pegl-17::NLS-cfp::LacZ + Pttx- } \\
\text { 3::gfp] V }\end{array}$ & Fig. S2A, C, D \\
\hline DV2750 & $\begin{array}{l}\text { let-60(n1046gf) IV; arls92[Pegl-17::NLS-cfp::LacZ + Pttx- } \\
\text { 3::gfp] V; rgl-1(tm2255) }\end{array}$ & Fig. S2B, E \\
\hline WU49 & lin-45(n2506rf) unc-24(e138) IV & $\begin{array}{l}\text { Fig. S2F, S5E, } \\
\text { S5F }\end{array}$ \\
\hline DV2783 & lin-45(n2506rf) unc-24(e138) IV; rgl-1(tm2255) X & Fig. S2F \\
\hline PS1524 & unc-4(e120) let-23(sa62gf) II & Fig. S2G \\
\hline DV2958 & unc-4(e120) let-23(sa62gf) II; rgl-1(tm2255) X & Fig. S2G \\
\hline BC14985 & $\begin{array}{l}\text { dpy-5(e907) I; sEx14985[rCesF28B4.2::gfp + PCeh361(dpy- } \\
5(+))]\end{array}$ & Fig. 3 \\
\hline DV3312 & rgl-1(re179[mNeonGreen^3xFlag::rgl-1]) X & Fig. S3 \\
\hline VC40420 & ral-1(gk628801rf) III (unoutcrossed) & Results \\
\hline DV2942 & ral-1(gk628801rf) III (6x outcrossed) & Results \\
\hline DV2799 & ral-1(gk628801) III; let-60(n1046gf) IV & Fig. 4C \\
\hline VC20011 & rgl-1(gk275304) X (unoutcrossed) & Results \\
\hline DV2883 & rgl-1(gk275304) $\times(4 \times$ outcrossed $)$ & Results \\
\hline VC40052 & rgl-1(gk275305) X (unoutcrossed) & Results \\
\hline DV2884 & rgl-1(gk275305) X (4x outcrossed $)$ & Results \\
\hline DV2764 & let-60(n1046gf) IV; rgl-1(gk275304)X & Fig. 4D \\
\hline DV2765 & let-60(n1046gf) IV; rgl-1(gk275305)X & Fig. 4D \\
\hline DV2537 & $\begin{array}{l}\text { let-60(n1046gf) IV; rgl-1(tm2255) X; reEx94[P } P_{\text {lin-31.: }}: \text { rgl- } \\
1(R 324 E)+P_{\text {mуо-2::GFP] }}\end{array}$ & Fig. 4E, S4A \\
\hline DV2538 & $\begin{array}{l}\text { let-60(n1046gf) IV; rgl-1(tm2255) X; reEx95[P } P_{\text {lin-31: }: \text { rgl- }} \\
\left.1(R 324 E)+P_{\text {mуо-2: }}: G F P\right]\end{array}$ & Fig. 4E, S4A \\
\hline DV2736 & $\begin{array}{l}\text { let-60(n1046gf) IV; rgl-1(tm2255) X; reEx109[P } P_{\text {lin-31: }: \text { rgl-1(+) + }} \\
P_{\text {myo-2::GFP] }}\end{array}$ & Fig. 4E, S4B \\
\hline
\end{tabular}




\begin{tabular}{|c|c|c|}
\hline DV2737 & 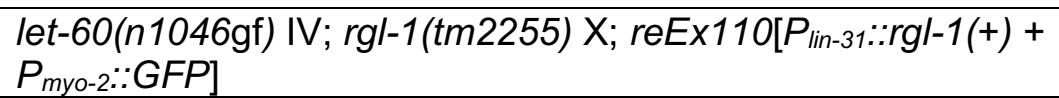 & Fig. 4E, S4B \\
\hline MT301 & lin-31(n301) II & Fig. S4C \\
\hline DV2763 & lin-31(n301) II; rgl-1(tm2255) & Fig. S4C \\
\hline DV2140 & let-60(n1046gf) IV; reEx24[Plin-31::ral-1(Q75L), $P_{\text {myo-2::gfp)] }}$ & $\begin{array}{l}\text { Zand, } 2011, \\
\text { derived rels10 }\end{array}$ \\
\hline DV2335 & 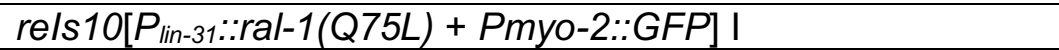 & Results \\
\hline DV2699 & $\begin{array}{l}\text { rels10[Plin-31::ral-1(Q75L) + Pmyo-2::GFP] I; let-60(n1046gf) } \\
\text { IV; let-60(n1046gf) IV }\end{array}$ & Fig. 4F, S4D \\
\hline DV2700 & $\begin{array}{l}\text { rels10[Plin-31::ral-1(Q75L) + Pmyo-2::GFP] I; let-60(n1046gf) } \\
\text { IV; let-60(n1046gf) IV; rgl-1(tm2255) X }\end{array}$ & Fig. 4F \\
\hline CB2065 & $d p y-11(\mathrm{e} 224)$ unc-76(e905) & akt-1 balancer \\
\hline GR1310 & akt-1(mg144gf) V & Fig. 5 \\
\hline DV2746 & let-60(n1046gf) IV; akt-1(mg144gf) V & Fig. 5A, S5A \\
\hline DV2747 & let-60(n1046gf) IV; akt-1 (mg144gf) V; rgl-1(tm2255) X & Fig. 5A, S5A \\
\hline GR1318 & $p d k-1(m g 142 g f) X$ & Fig. 5 \\
\hline DV2773 & let-60(n1046gf) IV; $p d k-1$ (mg142gf) X & Fig. 5B, S5B \\
\hline DV2782 & let-60(n1046gf) IV; $p d k-1$ (mg142gf) X & Fig. 5B \\
\hline DV2844 & let-60(n1046gf) IV; $p d k-1$ (mg142gf) X & Fig. 5B \\
\hline SP934 & unc-1(e538) dpy-3(e27) X & Fig. $5 \mathrm{~B}$ \\
\hline DV2774 & unc-1(e538) dpy-3(e27) rgl-1(tm2255) X & Fig. 5B \\
\hline DV2819 & $p d k-1$ (mg142gf) rgl-1(tm2255) X & Fig. $5 \mathrm{~B}$ \\
\hline DV2822 & let-60(n1046gf) IV; $p d k-1$ (mg142gf) rgl-1(tm2255) X & Fig. 5B \\
\hline DV2791 & lin-45(n2506rf) unc-24(e138) IV; akt-1(mg144gf) V & Fig. S5F \\
\hline DV2795 & lin-45(n2506rf) unc-24(e138) IV; pdk-1(mg142gf) X & Fig. S5E \\
\hline RB712 & daf-18(ok480) IV & Starting reagent \\
\hline DV2855 & daf-18(ok480) 2x outcrossed & Fig. 5 \\
\hline DV2510 & daf-18(ok480) let-60(n1046gf) IV & $\begin{array}{l}\text { Fig. 5C, D, S5C, } \\
\text { S5D, S5G, S5H }\end{array}$ \\
\hline DV2535 & daf-18(ok480) let-60(n1046gf) IV; rgl-1(tm2255) X & Fig. 5C \\
\hline DV2509 & daf-18(ok480) let-60(n1046gf) IV; rgl-1(tm2255) X & Fig. 5D \\
\hline DV2679 & jip-1(km18) II & Fig. S5I \\
\hline DV3013 & jip-1(km18) II; let-60(n1046gf) IV & Fig. S5I \\
\hline DV3358 & jip-1(tm6137) II (4x outcrossed) & Fig. S5I \\
\hline DV3505 & jip-1(tm6137) II; let-60(n1046gf) IV & Fig. S5I \\
\hline DV3506 & jip-1(tm6137) II; daf-18(ok480) let-60(n1046gf) IV & Fig. S5I \\
\hline DV2696 & rgl-1(ok1921) 8x outcrossed & Fig. 6 \\
\hline DV2697 & rgl-1(tm2255) 8x outcrossed & Fig. 6 \\
\hline
\end{tabular}




\section{Supplementary Table 2 - Primers}

\begin{tabular}{|c|c|c|}
\hline Name & Sequence & Use \\
\hline DJR614 & 5'-GAGCAACTGACGTTTTGGGATGC-3' & $\begin{array}{l}\text { rgl- } \\
1 \text { (ok1921) } \\
\text { genotyping }\end{array}$ \\
\hline DJR615 & 5'-GATCTGGAGTGGAGTGCATTGG-3' & $\begin{array}{l}\text { rgl- } \\
1 \text { (ok1921) } \\
\text { genotyping }\end{array}$ \\
\hline DJR616 & 5'-CGAAAAGCTCCCCACTTCGACG-3' & $\begin{array}{l}\text { rgl- } \\
\text { 1(ok1921) } \\
\text { genotyping }\end{array}$ \\
\hline DJR778 & 5'-TAGACAATTTAGGCCCAAAACCCCCG-3' & $\begin{array}{l}\text { ral- } \\
\text { 1(gk628801 } \\
\text { ) genotyping }\end{array}$ \\
\hline DJR779 & 5'-CCAAATTTTCAGCCTAAAATCTCTTCCCAATACC-3' & $\begin{array}{l}\text { ral- } \\
\text { 1(gk628801 } \\
\text { ) genotyping }\end{array}$ \\
\hline RGL-1F & $\begin{array}{l}\text { 5'-GCGGGATCCGAAAAAATGGCTACGCGTTACTGGGGTGACG- } \\
\text { 3' }\end{array}$ & $\begin{array}{l}\text { Cloning rgl- } \\
\text { 1a cDNA }\end{array}$ \\
\hline RGL-1R & 5'-ATAAGAATGCGGCCGCTTTACAAGTAGCCACTGCTCCATG-3' & $\begin{array}{l}\text { Cloning rgl- } \\
\text { 1a cDNA }\end{array}$ \\
\hline DRC1 & 5'-AGATGCTAGCTGACGGAGATGTGGG-3' & $\begin{array}{l}\text { pdk- } \\
1 \text { (mg142gf) } \\
\text { genotyping }\end{array}$ \\
\hline DRC2 & 5'-AAATGTGGCTGGAATGTAGGCGTGC-3' & $\begin{array}{l}\text { pdk- } \\
1 \text { (mg142gf) } \\
\text { genotyping }\end{array}$ \\
\hline FSM4 & 5'-ATATTCGAGGAGTCGGTGGTCC-3' & $\begin{array}{l}\text { daf- } \\
\text { 18(ok480) } \\
\text { genotyping }\end{array}$ \\
\hline FSM5 & 5'-GAGGCTACCGGATAATGTGC-3' & $\begin{array}{l}\text { daf- } \\
\text { 18(ok480) } \\
\text { genotyping }\end{array}$ \\
\hline FSM6 & 5'-GGCAACGAATGAATACGCAGG-3' & $\begin{array}{l}\text { daf- } \\
\text { 18(ok480) } \\
\text { genotyping }\end{array}$ \\
\hline FSM7 & 5'-GAAGTCAAGCCGCTCTTCC-3' & $\begin{array}{l}\text { rgl- } \\
1 \text { (tm2255) } \\
\text { genotyping }\end{array}$ \\
\hline FSM8 & 5'-GGAGAACTGCTGGAGAACG-3' & $\begin{array}{l}\text { rgl- } \\
\text { 1(tm2255) } \\
\text { genotyping }\end{array}$ \\
\hline FSM9 & 5'-CCGTTCCCTGACATTCGG-3' & $\begin{array}{l}\text { rgl- } \\
1(\operatorname{tm} 2255) \\
\text { genotyping }\end{array}$ \\
\hline HS160 & $\begin{array}{l}\text { 5'- } \\
\text { ACACCTTCGTATCCTTGTGGGTTTAAGAGCTATGCTGGAAACAG } \\
-3 \text { ' }\end{array}$ & $\begin{array}{l}\text { rgl-1 sgRNA } \\
\text { Cas-9 } \\
\text { plasmid \#1 }\end{array}$ \\
\hline
\end{tabular}




\begin{tabular}{|c|c|c|}
\hline HS161 & $\begin{array}{l}\text { 5'- } \\
\text { GGTCTGAGTTCTTCTGACGAGTTTAAGAGCTATGCTGGAAACA } \\
\text { G-3' }\end{array}$ & $\begin{array}{l}\text { sgRNA } \\
\text { Cas-9 } \\
\text { plasmid \#2 }\end{array}$ \\
\hline DJR769 & 5'-CACCTCCTATTGCGAGATGTCTTG-3' & $\begin{array}{l}\text { Universal } \\
\text { sgRNA } \\
\text { plasmid } \\
\text { Mutagenesi } \\
\text { s }\end{array}$ \\
\hline HS120 & $\begin{array}{l}\text { 5'- } \\
\text { CCAGTCACGACGTTGTAAAACGACGGCCAGTCGCCGGCACCG } \\
\text { CATCCATTCACAGTGTAC-3' }\end{array}$ & $\begin{array}{l}\text { Left } \\
\text { homology } \\
\text { arm }\end{array}$ \\
\hline HS159 & $\begin{array}{l}\text { 5'- } \\
\text { AGGGAGGCCATGTTGTCCTCCTCTCCCTTGGAGACCATACGCG } \\
\text { TAGCCATTTAAGATTGGTCTGAGTTCTTCTGACG-3' }\end{array}$ & $\begin{array}{l}\text { Left } \\
\text { homology } \\
\text { arm }\end{array}$ \\
\hline HS122 & $\begin{array}{l}\text { 5'- } \\
\text { CGACGACAAGCGTGATTACAAGGATGACGATGACAAGAGAGGA } \\
\text { TCTGGAATGGCTACGCGTTACTGGG-3' }\end{array}$ & $\begin{array}{l}\text { Right } \\
\text { homology } \\
\text { arm }\end{array}$ \\
\hline HS123 & $\begin{array}{l}\text { 5'- } \\
\text { TAACAATTTCACACAGGAAACAGCTATGACCATGTTATCGAAGT } \\
\text { GGCACTCAGCTTCATC-3' }\end{array}$ & $\begin{array}{l}\text { Right } \\
\text { homology } \\
\text { arm }\end{array}$ \\
\hline HS125 & 5'-CTTGTCACTGTAAGGGAAGATTTCC3'- & $\begin{array}{l}\text { rgl-1 } \\
\text { CRISPR } \\
\text { genotyping }\end{array}$ \\
\hline HS126 & 5'-TTGTCCTCCTCTCCCTTGG-3' & $\begin{array}{l}\text { rgl-1 } \\
\text { CRISPR } \\
\text { genotyping }\end{array}$ \\
\hline HS127 & 5’ ACGTAGAATGTTCCAGAGTTCCAG-3 & $\begin{array}{l}\text { rgl-1 } \\
\text { CRISPR } \\
\text { genotyping }\end{array}$ \\
\hline KM1 & $\begin{array}{l}\text { 5'- } \\
\text { GCCGGCCCGAATACGAAGCGAAGATAATCAGCAAGTGGATCG- } \\
\text { 3' }\end{array}$ & $\begin{array}{l}\text { R324E } \\
\text { mutagenesi } \\
\text { s }\end{array}$ \\
\hline KM2 & 5'-GATCCACTTGCTGATTATCTTCGCTTCGTATTCGGGCCGG-3' & $\begin{array}{l}\text { R324E } \\
\text { mutagenesi } \\
\text { s }\end{array}$ \\
\hline
\end{tabular}


A

(AC)
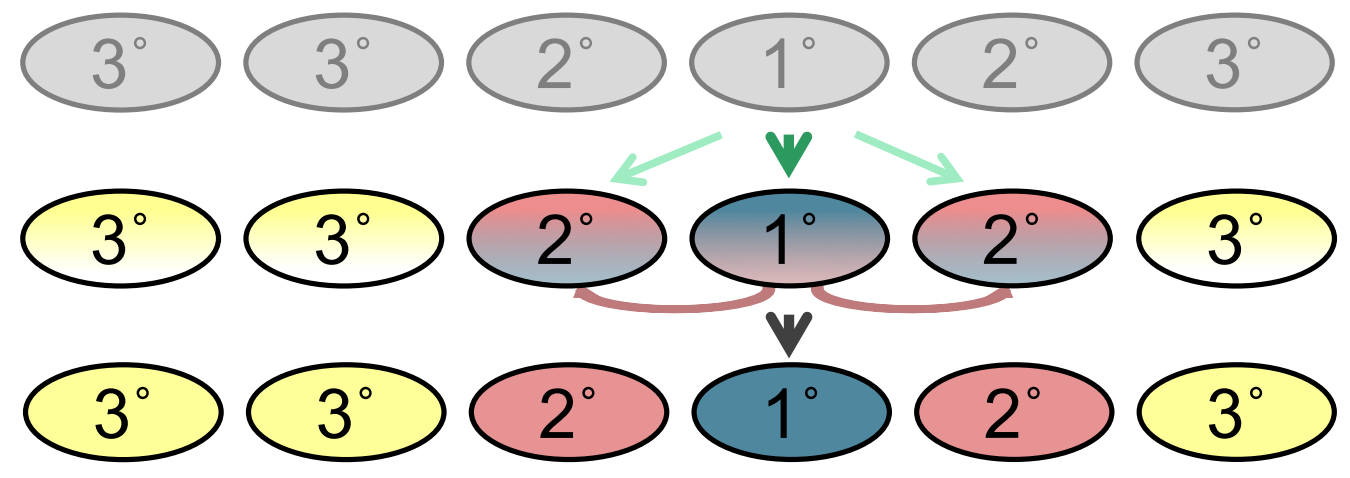

B EGF

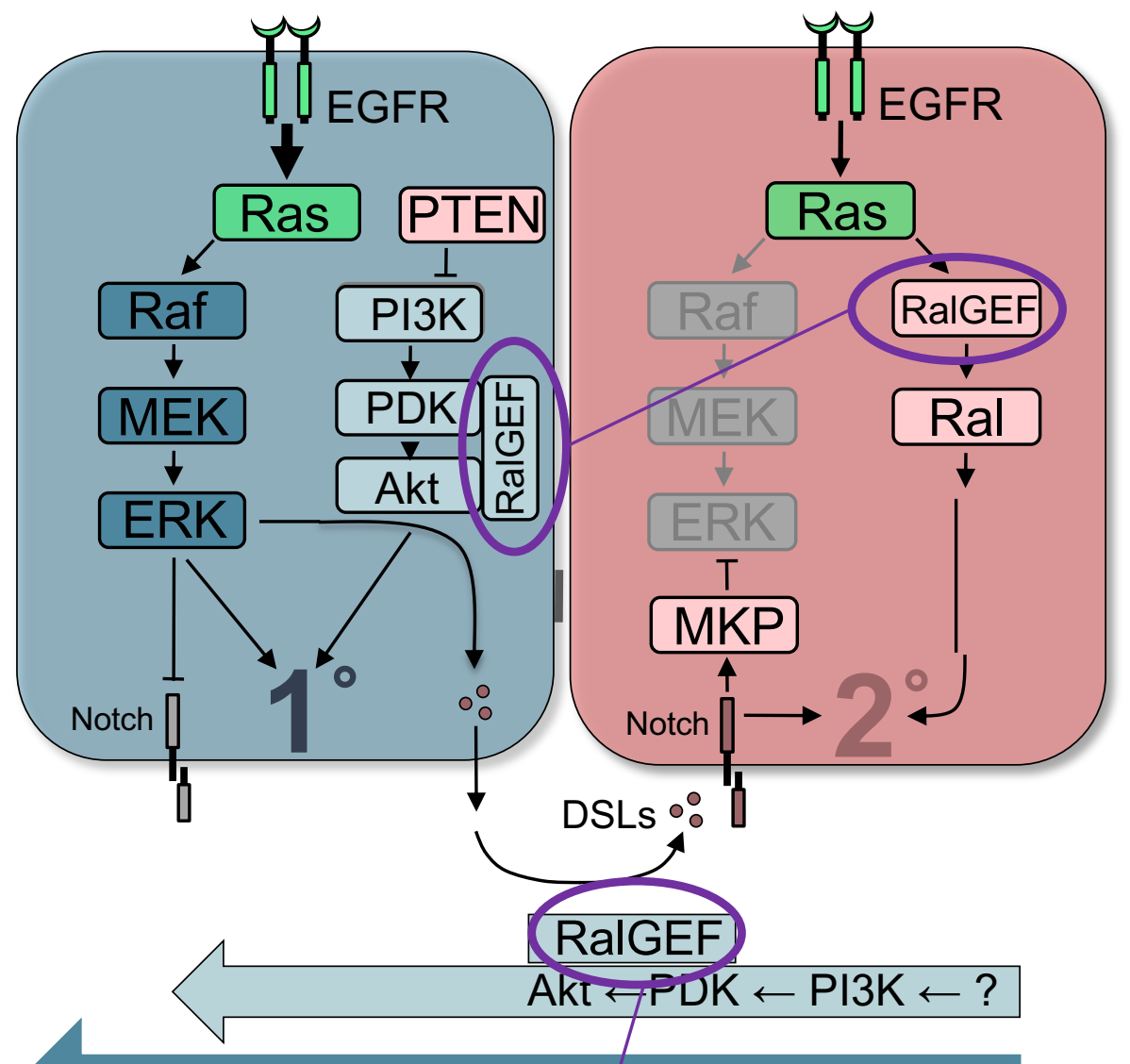

C

RalGEF

Akt $F U K \leftarrow P I 3 K \leftarrow ?$

$\mathrm{ERK} \leftarrow \mathrm{MEK} \leftarrow$ Raf $\leftarrow$ Ras $\leftarrow$ EGFR

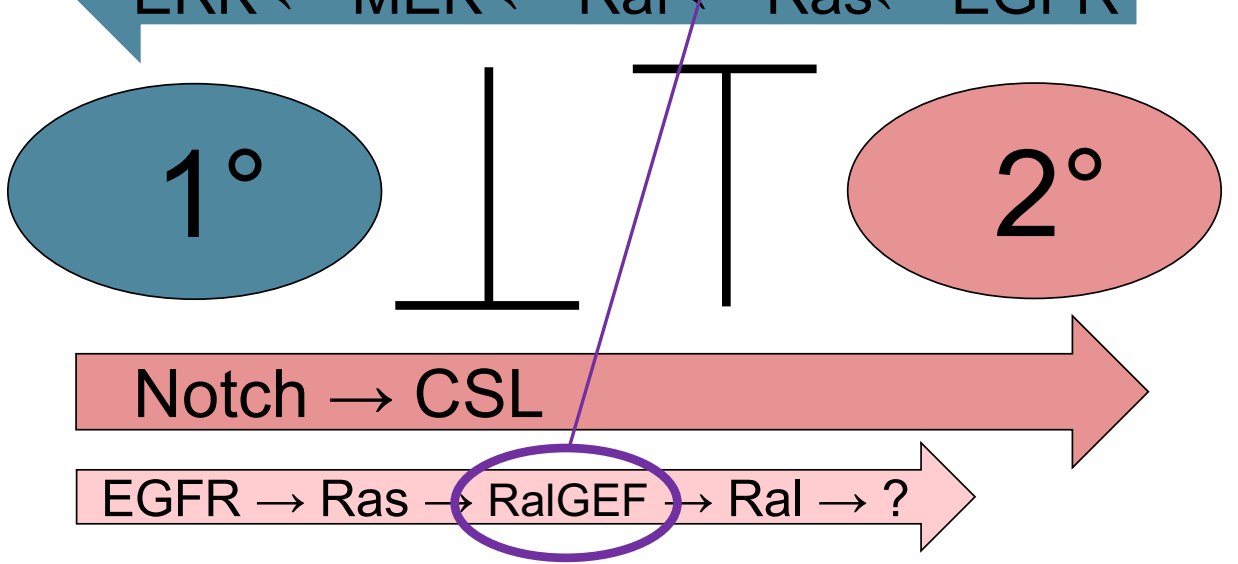

Shin Fig. 1 

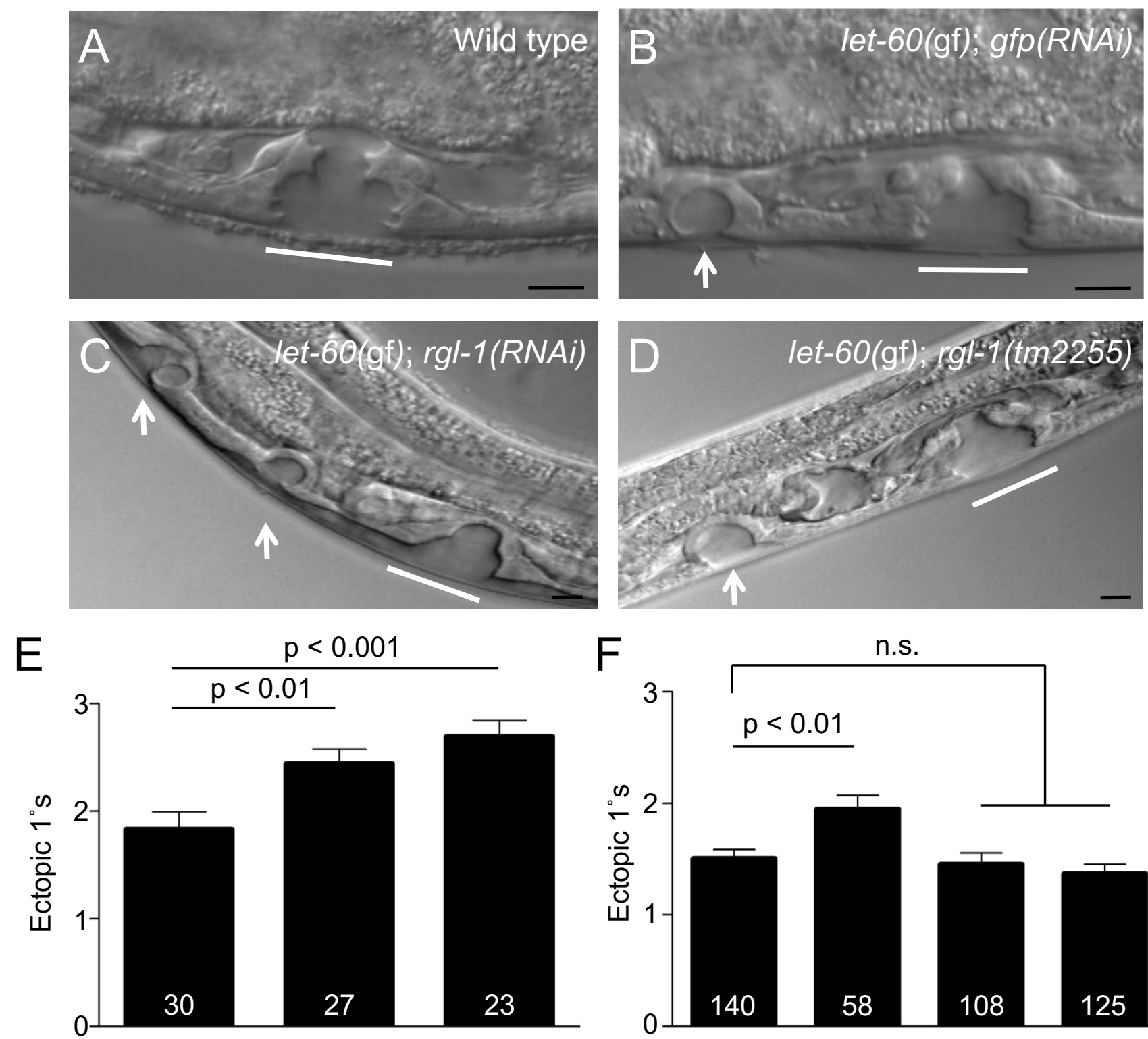

F

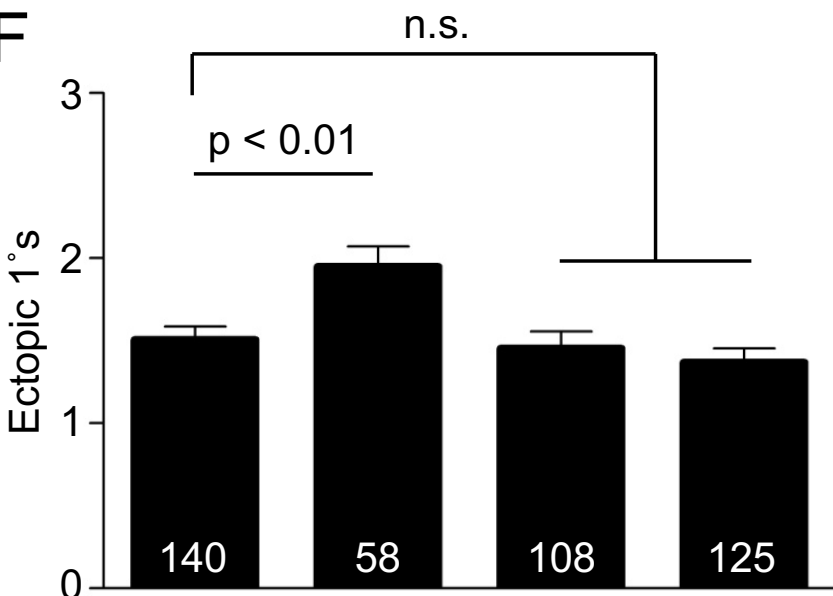

Background: let-60(n1046gf) RNAi: $g f p \quad r a l-1 \quad r g l-1 \quad$ Mutation: + Background: let-60(n1046gf)

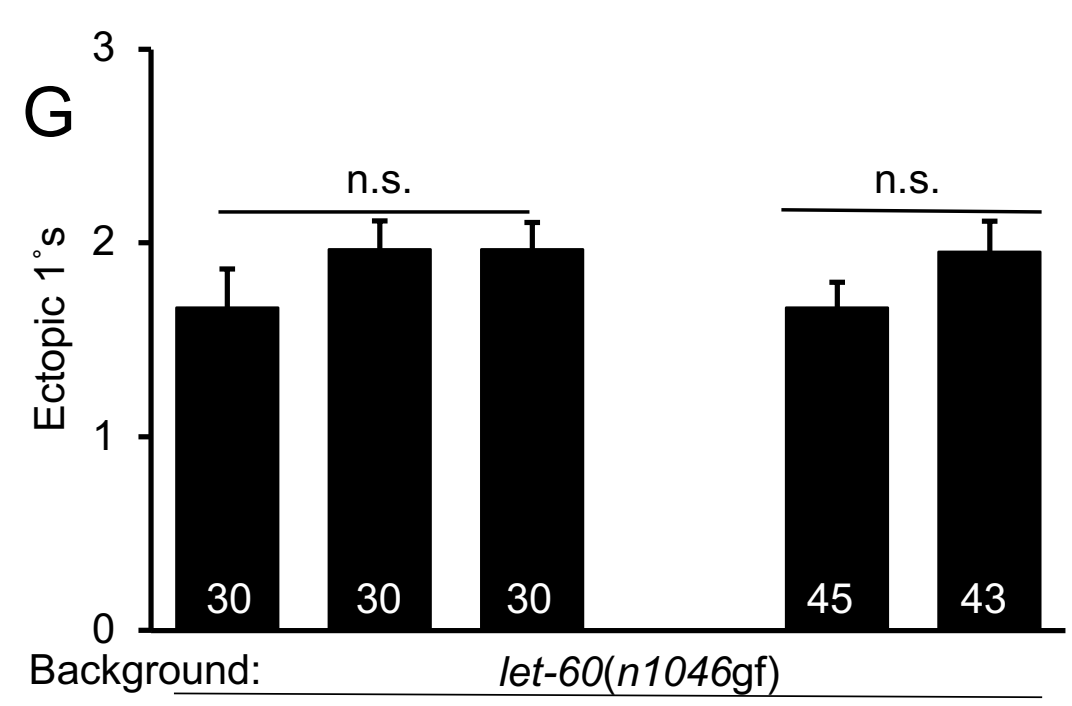

rgl-1: + ok1921 tm2255 $\frac{+ \text { gm27 }}{\text { lon-2 }}$

$\mathrm{H}$

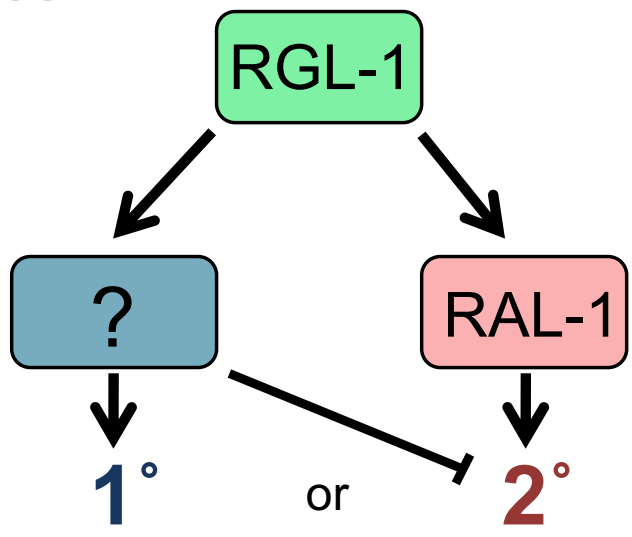

Shin Fig. 2 


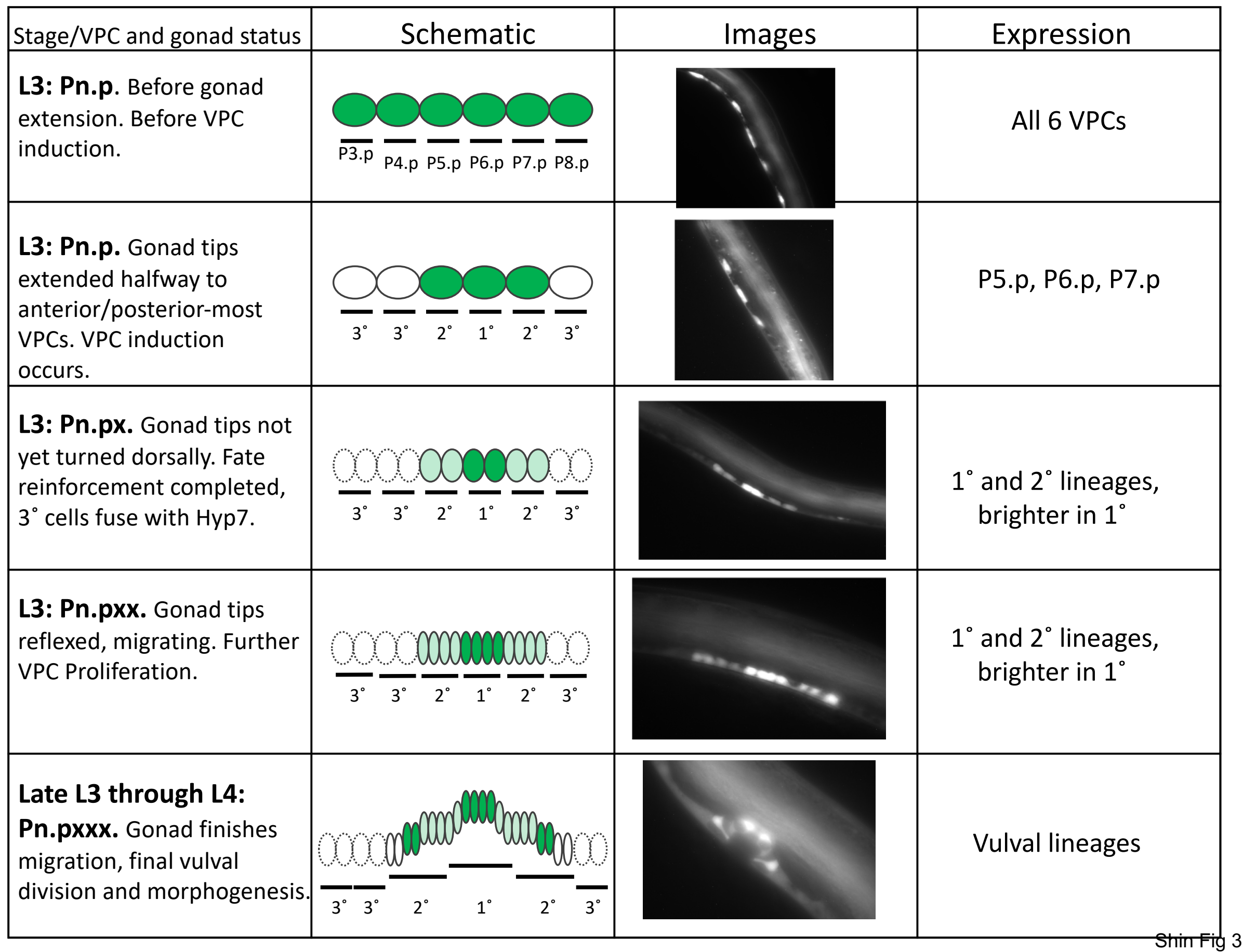



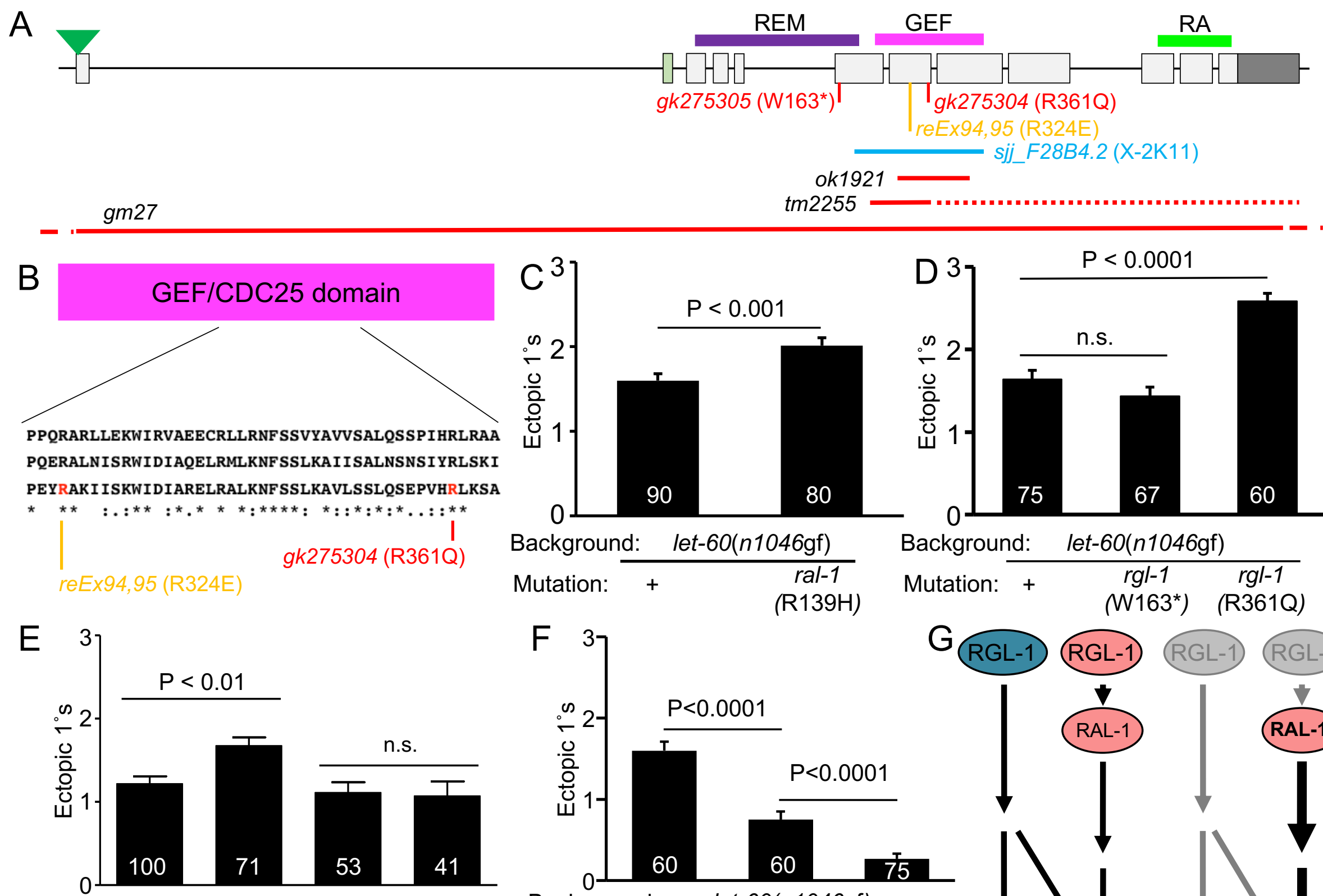

Background: let-60(n1046gf); rgl-1(tm2255)

Transgene: - R324E $\frac{-}{\text { sibs }} \stackrel{+}{\text { sibs }}$
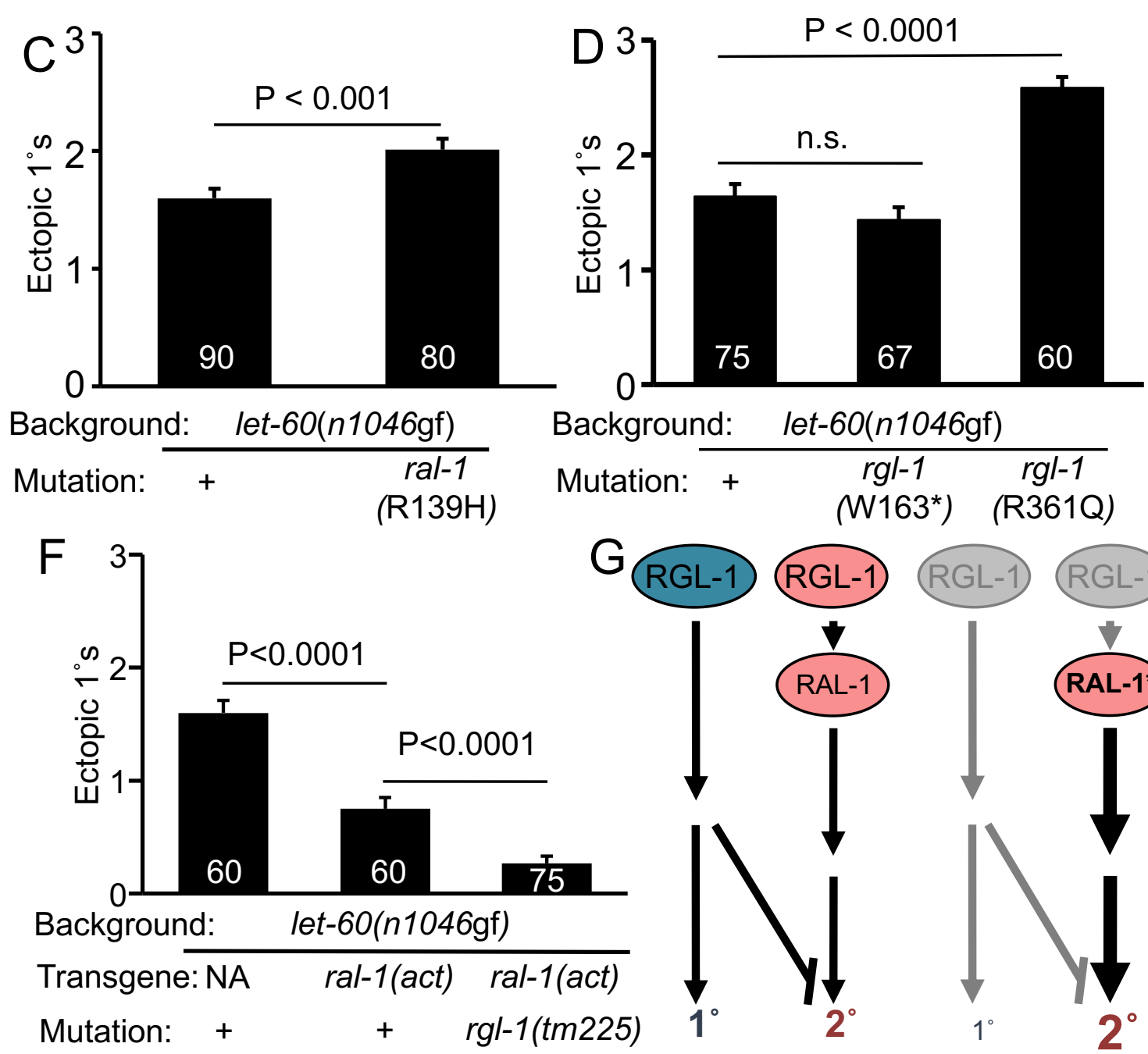

(W163*) (R361Q)

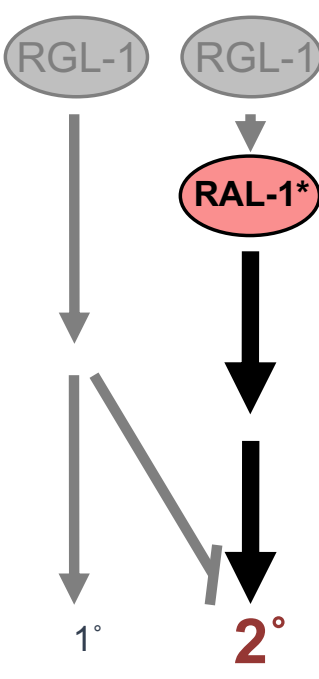

Shin Fig. 4 

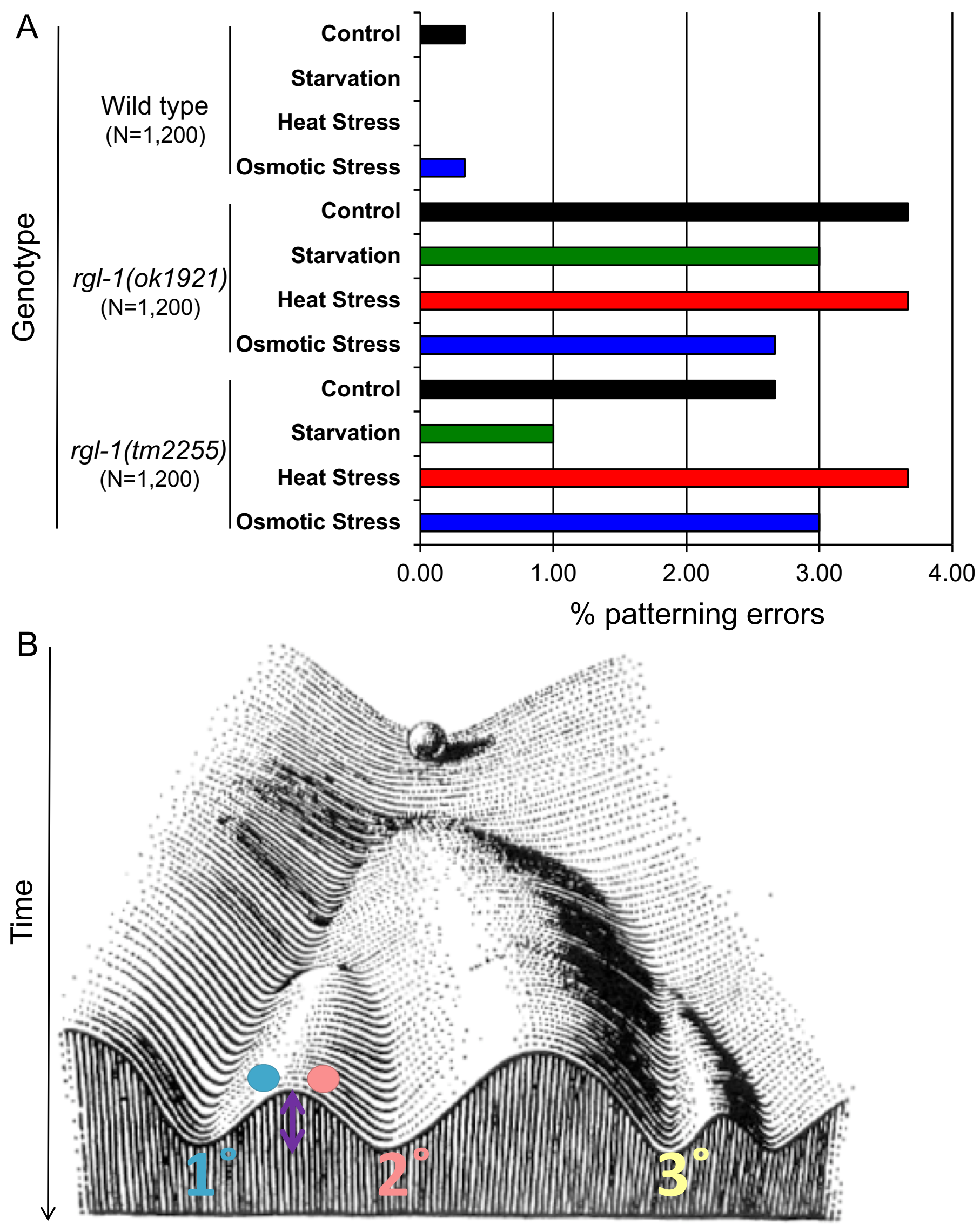

RGL-1/RalGEF (Ral/Akt) circuit

Shin Fig. 6 


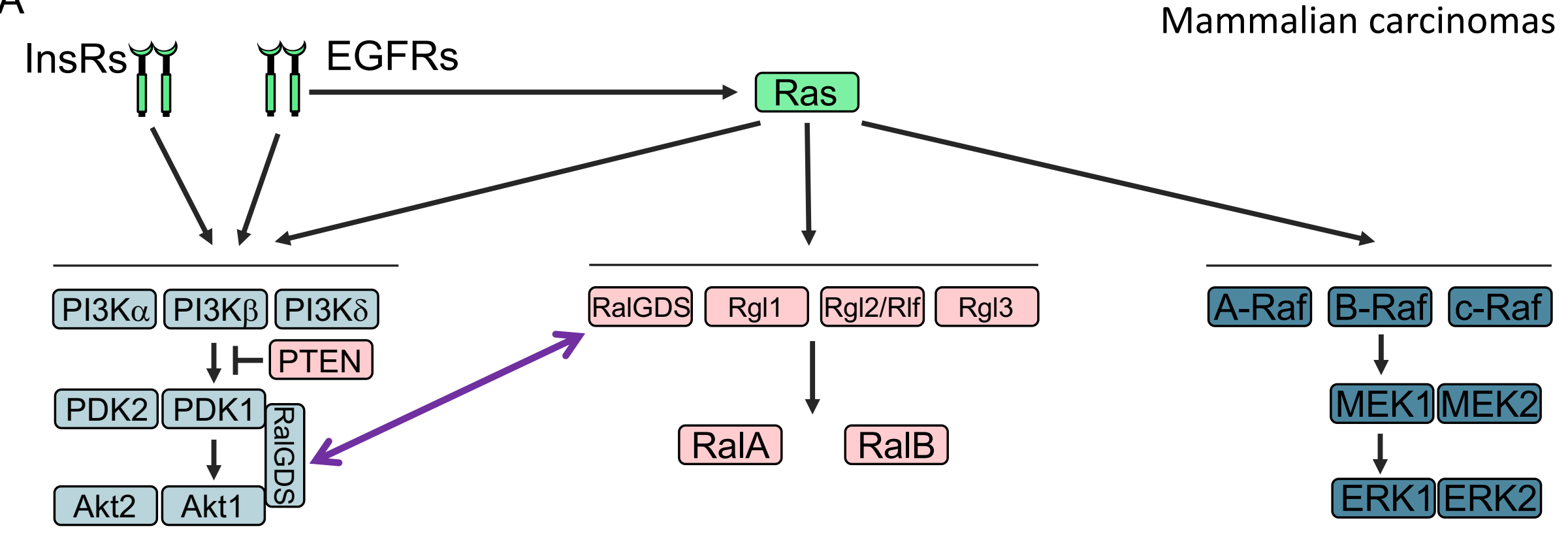

B

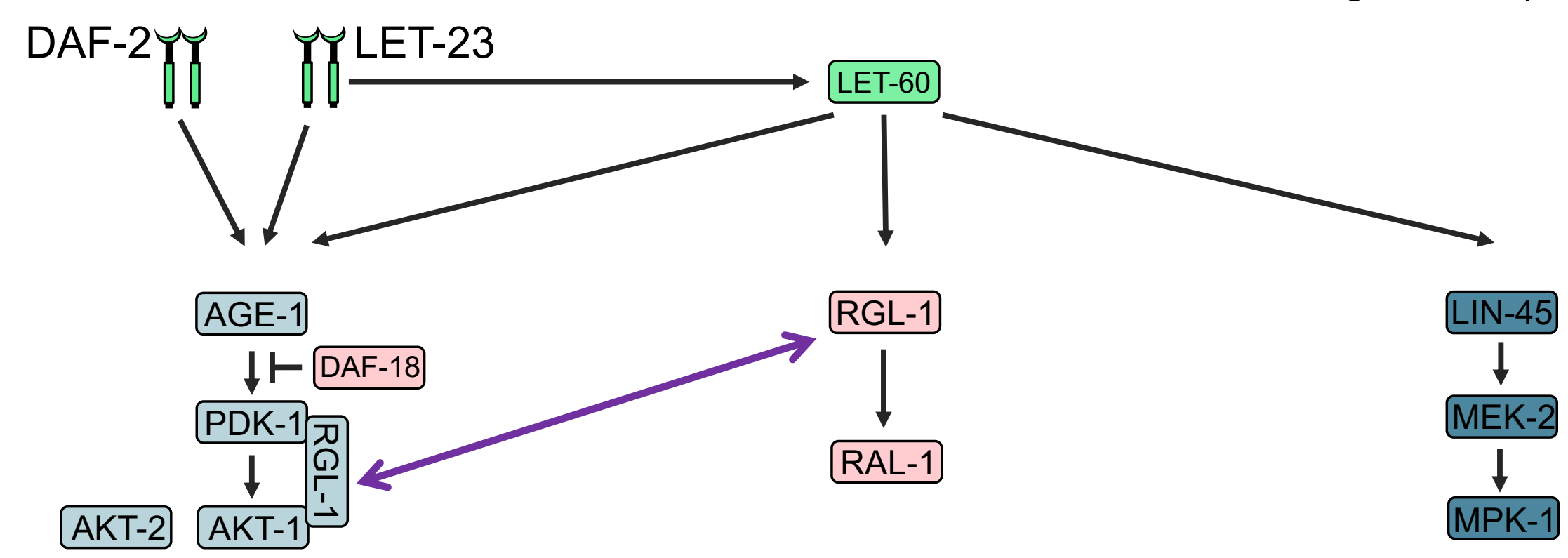

Shin Fig. S1 
Figure S1. Signaling network comparisons between humans and C. elegans. Signaling relationships in A) Mammalian carcinomas and B) C. elegans VPC fate patterning. Historically, mammalian interactions have been shown directly, while $C$. elegans interactions were deduced from a combination of phenotypes, genetic epistasis, and inferences from biochemical relationships among mammalian orthologs. Color coding is the same as in other figures: blue $=1^{\circ}$-promoting, rose $=2^{\circ}$-promoter, dark $=$ necessary and sufficient signal, light $=$ modulatory signal. Green $=$ both $1^{\circ}-$ and $2^{\circ}$-promoting $($ rather than green, RGL-1 is shown in two places, with a purple two-headed arrow denoting possible dual function in both non-canonical $1^{\circ}$-promoting and canonical $2^{\circ}$ promoting roles). Activation of $C$. elegans AGE-1/PI3K by a receptor other than DAF-2, or by LET-60/Ras, has not been suggested in the literature. The interactions between RGL-1, PDK-1 and AKT-1 are inferred from genetic relationships in this study, and have not been shown directly. JIP-1, a potential intermediary between Akt and RalGDS/RalGEFs inferred from mammalian biochemical analyses in the Feig lab, is not shown. 

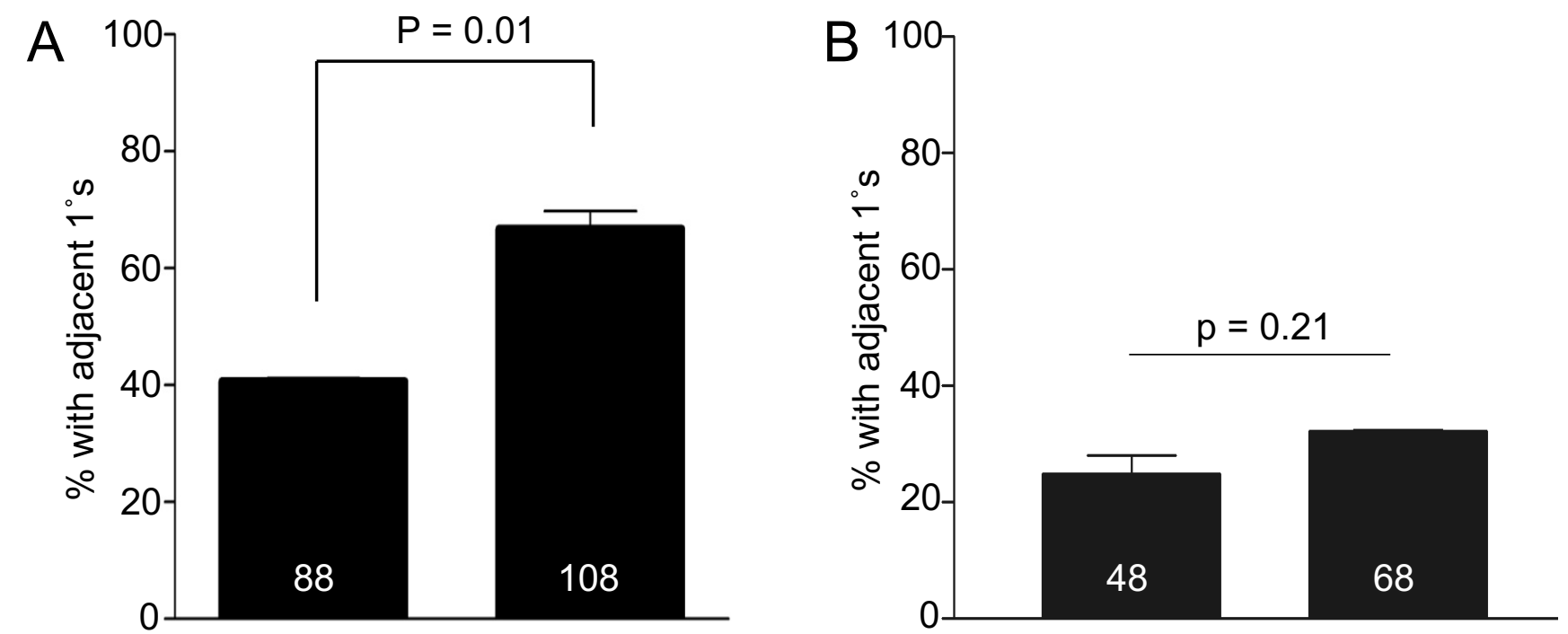

Genotype: arls92; let-60(n1046gf)

Genotype: $\quad$ arls92; let-60(n1046gf)

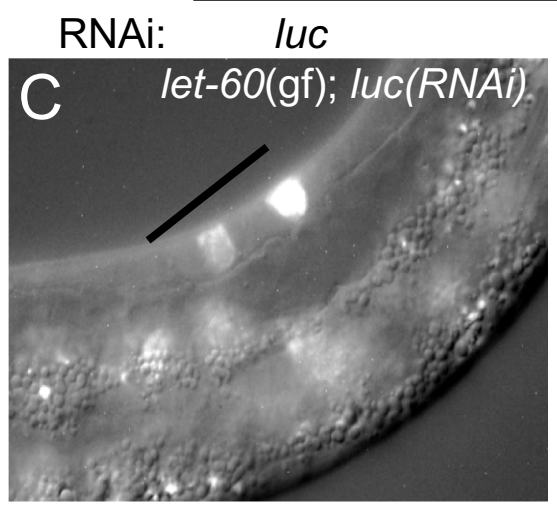

rgl-1

Deletion:

rgl-1(+) rgl-1(tm2255)
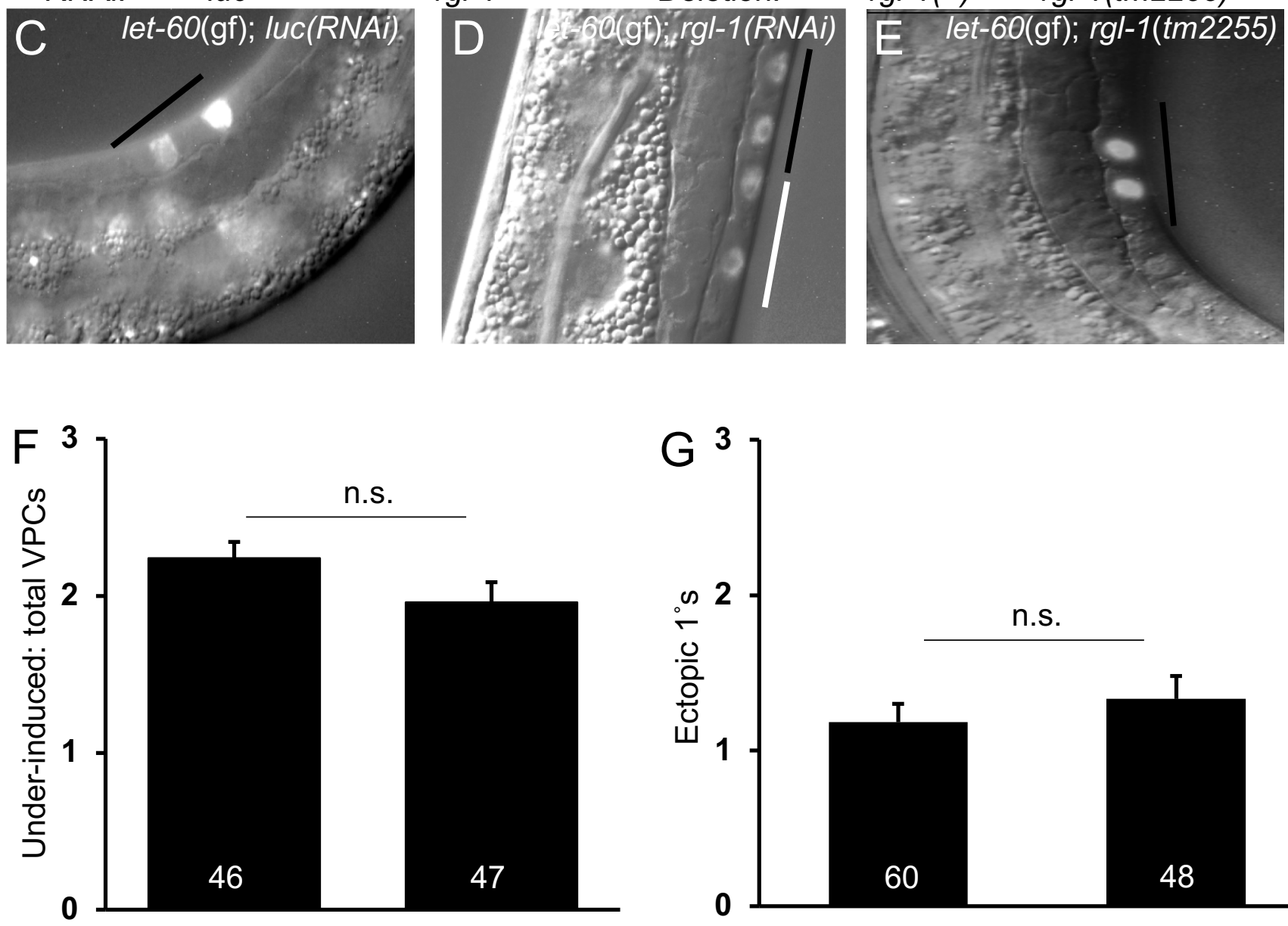

Genotype:

lin-45(n2506rf)

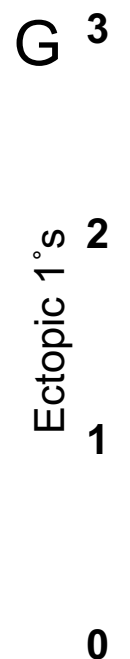

Genotype:

n.s.

Deletion: $r g l-1(+) \quad$ rgl-1(tm2255)

Deletion: $\quad r g l-1(+) \quad r g l-1(t m 2255)$ 
Figure S2: RGL-1 controls cell fate decision. A) Percent Pn.px-staged let-60(gf) L3 larvae with CFP-positive lineages neighboring the P6.p lineage (P5.p or P7.p derived) with luc(RNAi) vs. rgl1(RNAi). Shown are average percentages of animals with adjacent $1^{\circ}$ cell fate. B) Percent Pn.pxstaged let-60(gf) L3 larvae with CFP-positive lineages neighboring the P6.p lineage (P5.p or P7.p derived) with $\mathrm{rgl}-1(+)$ or $\mathrm{rgl}-1(\operatorname{tm} 2255 \Delta)$. $\mathrm{Y}$ axis is percent adjacent $1^{\circ} \mathrm{s}$, white numbers in bars are number of animals scored per genotype. C-E) Expression of the $1^{\circ}$ fate reporter arls92 $\mathrm{P}_{\text {egl-17 }}:$ :cfplacZ in VPC daughters. Overlaid DIC and CFP fluorescence images of C) let-60(n1046gf); luc(RNAi), D) let-60(n1046gf); rgl-1(RNAi) and E) let-60(The black bar indicates P6.px and white bar indicates P7.px cells. n1046gf); rgl-1(tm2255A) at the Pn.px stage. F) Hypo-induced lin45(n2506) background with and without tm2255. $Y$ axis is total induced VPCs $(0=$ vulvaless, $3=$ normal wild-type vulva.) White numbers are number of animals assayed. G) let-23(sa62gf) with and without tm2255. $\mathrm{Y}$ axis is mean ectopic $1^{\circ}$ induction. Error bars show S.E.M. P value calculated via Mann-Whitney test. 


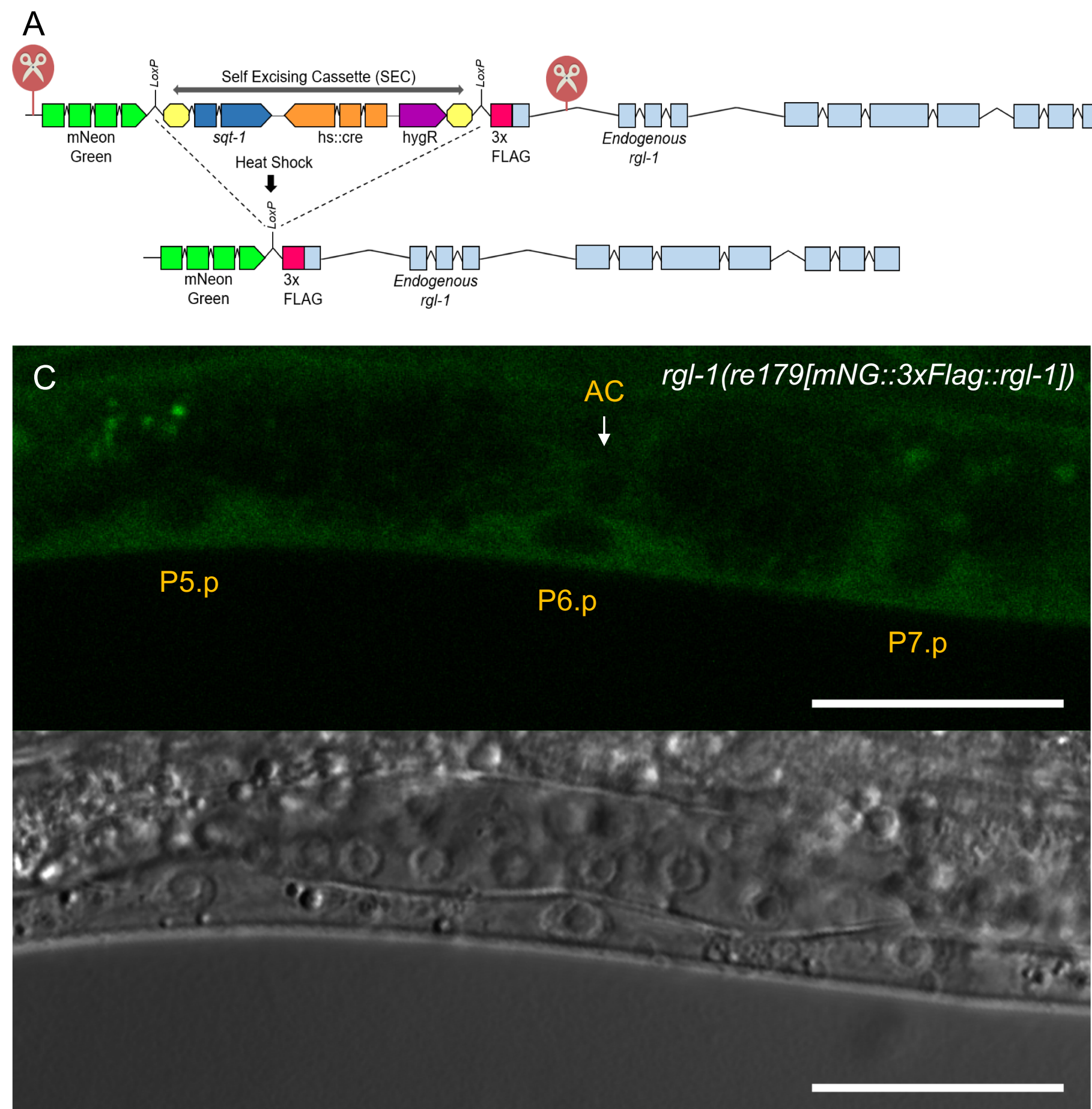

B

$150 \mathrm{kDa}-$
$100 \mathrm{kDa}-$

$50 \mathrm{kDa}$ 
Figure S3. N-terminal CRISPR tagging of endogenously expressed RGL-1 protein. A) Strategy for N-terminal tagging of the endogenous RGL-1 protein, using the SEC approach (Dickinson, 2015). See Methods. B) Western blot validation of RGL-1 N-terminal tag prior to (lane 1) and after SEC excision (lane 2). Below: alpha-tubulin loading control. C) Tagged endogenous mNG::RGL-1 is expressed in all VPCs. Scale bar $=20 \mu \mathrm{m}$. 
A

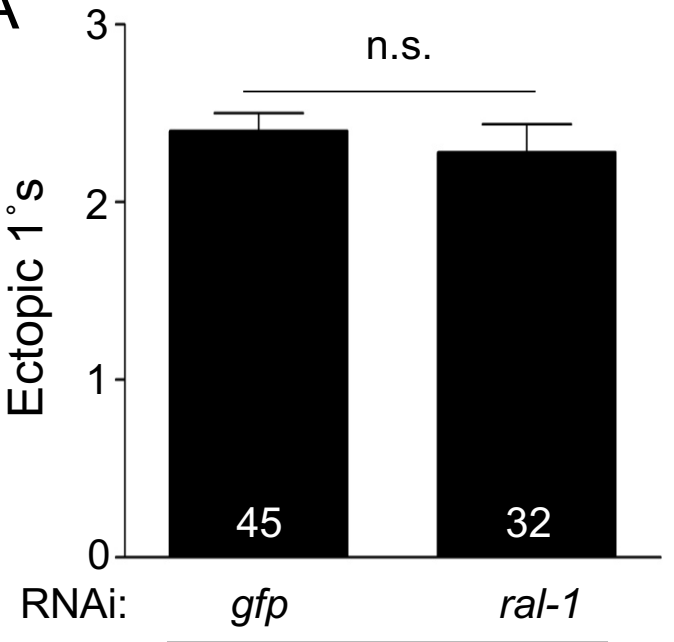

Genotype: let-60(gf); rgl-1(tm2255) + reEx94[rgl-1(R326E)]

C

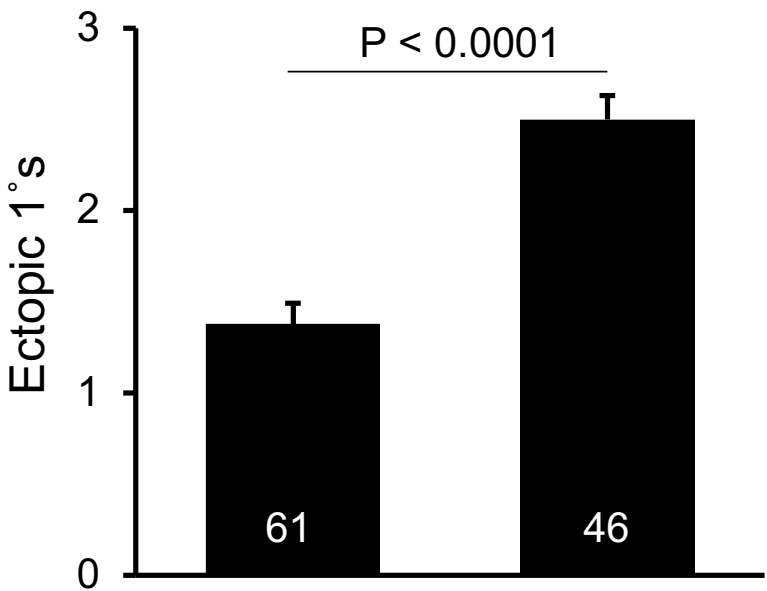

Genotype: lin-31
B

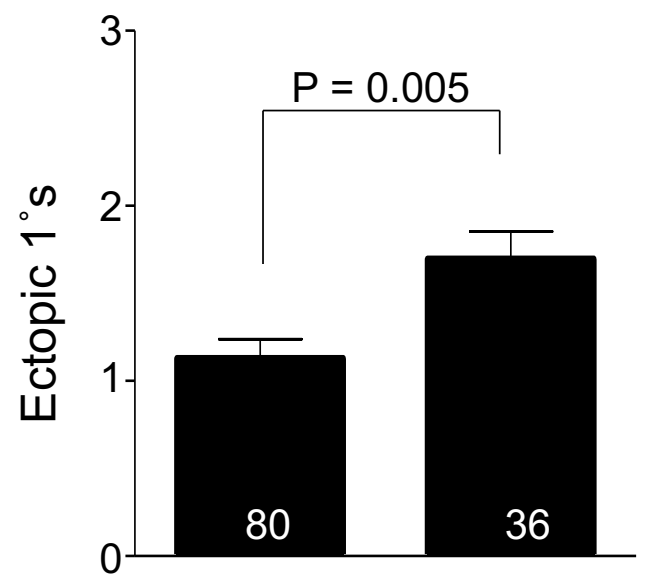

RNAi:

gfp

ral-1

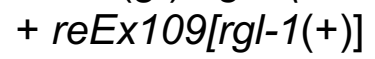
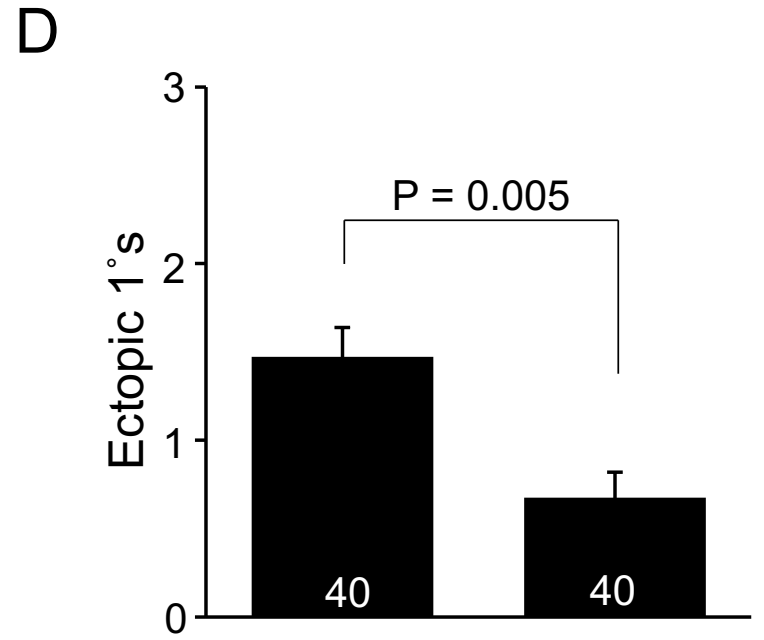

Genotype: let-60(gf) rels10[ral-1(gf)]; let-60(gf)

Figure S4. Genetically separable functions of RGL-1. A) let-60(gf); rgl-1(tm2255) animals rescued by VPCspecific expression of GEF dead (R326E) RGL-1 fail to respond to ral-1-directed vs. control GFP RNAi. B) let60(gf); rgl-1(tm2255) animals rescued by VPC-specific expression of wild-type RGL-1 rescue responsiveness to ral-1-directed but not control GFP RNAi. C) lin-31(n301) bypassed the putative $1^{\circ}$-promoting but not the putative $2^{\circ}$-promoting activity of $\mathrm{rgl}-1$, revealed by the $t m 2255$ mutation enhancing ectopic $1^{\circ}$ induction. D)

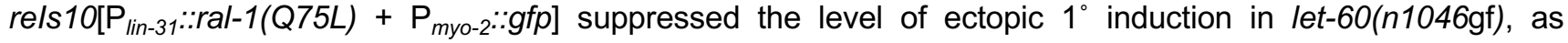
previously described for reEx24 (Zand, 2011). Y axis represents mean ectopic $1^{\circ}$ cells, white labels number of animals counted, error bars show S.E.M. P value calculated via Mann-Whitney test. 


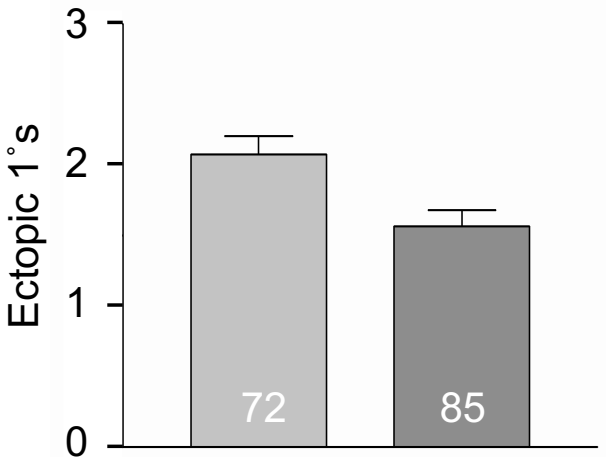

RNAi: $g f p$ jip-1

Background: daf-18(ok480) let-60(gf)

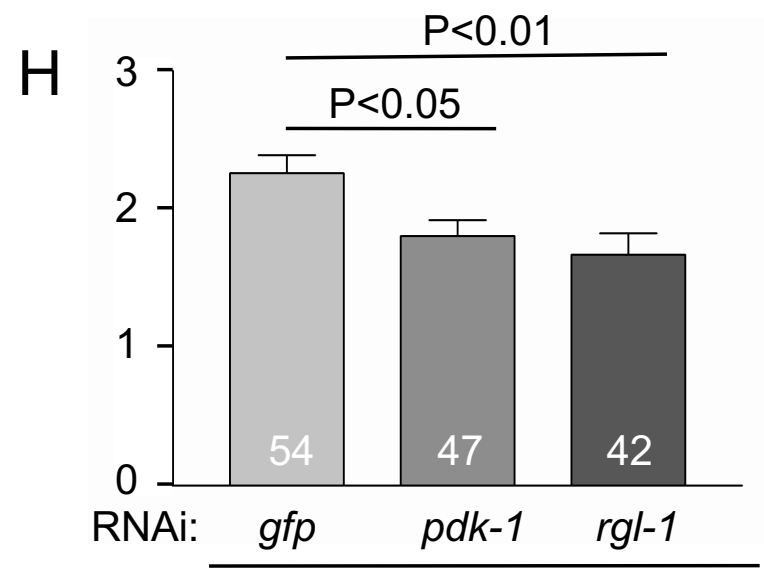

Background: daf-18(ok480) let-60(gf)
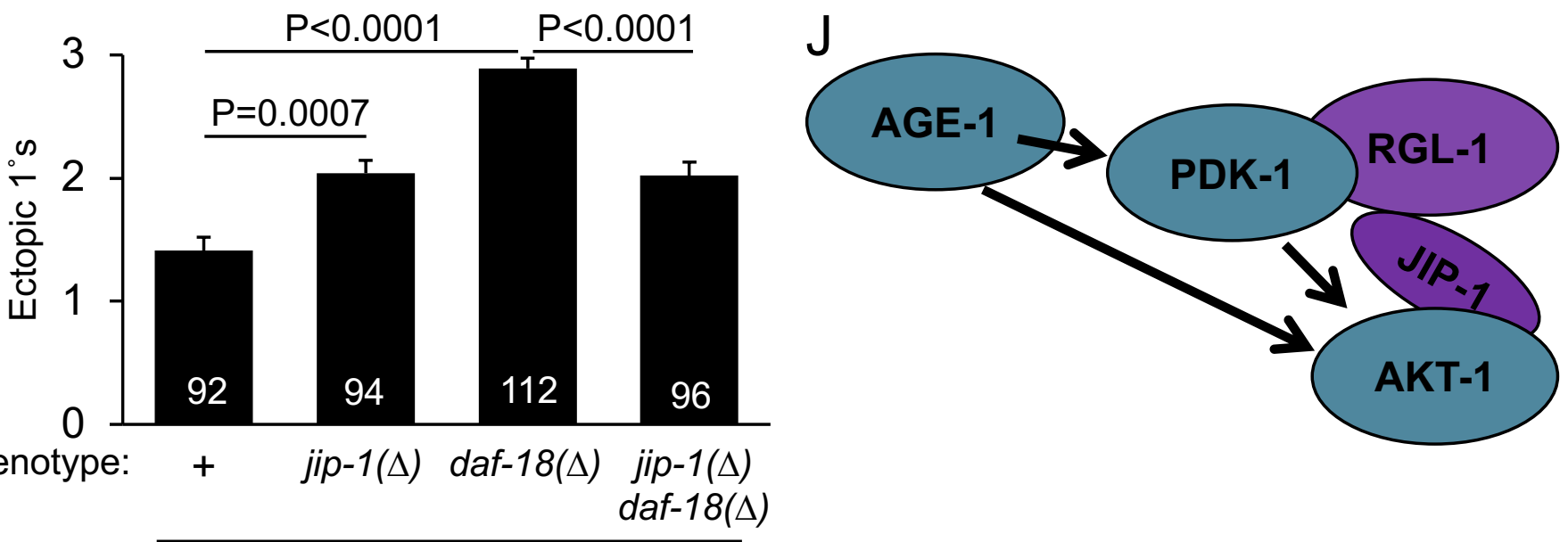

Background:

let-60(gf)

Figure 5: RGL-1 interacts genetically with the $1^{\circ}$-promoting AGE-1/PI3K-PDK-1-AKT-1 cascade. akt1(mg144gf) enhances $1^{\circ}$ induction in let-60(n1046gf) animals, and this enhancement is blocked by A) rgl1(tm2255) and B) rgl-1(ok1921), though not to the original baseline. The lin-45(n2506) under-induced mutant is partially suppressed by C) pdk-1(mg142gf) not D) akt-1(mg144gf). Unlike other assays, data shown are total induced VPCs, not ectopic $1^{\circ} \mathrm{s}$. daf-18(ok480) enhances $1^{\circ}$ induction in let-60(n1046gf) animals, and this enhancement is blocked by the E) rgl-1(gk275305) nonsense mutation but not the F) rgl-1(gk275304) R361Q putative GEF dead mutation. G) jip-1-directed RNAi suppressed the increase in ectopic $1^{\circ}$ induction in the let60(n1046gf) background conferred by daf-18(ok480), compared to gfp(RNAi). H) pdk-1- and rgl-1-directed RNAi similarly suppress daf-18(ok480) let-60(n1046gf) ectopic $1^{\circ}$ induction. I) The jip-1 deletion mutation, jip1(tm6137), enhances let-60(n1046gf) alone but suppresses daf-18(ok480) let-60(n1046gf), consistent with JIP-1 performing two functions. J) The observed genetic interactions are consistent with RGL-1 and JIP-1 functioning in the AGE-1-PDK-1-AKT-1 $1^{\circ}$ promoting cascade, as described for mammalian orthologs (Tian et al., 2002; Hao Wong and Feig, 2008). Except for lin-45(rf), Y axis represents mean ectopic $1^{\circ}$ cells, white labels number of animals counted, error bars show S.E.M. P value calculated via Mann-Whitney test or ANOVA. 
A
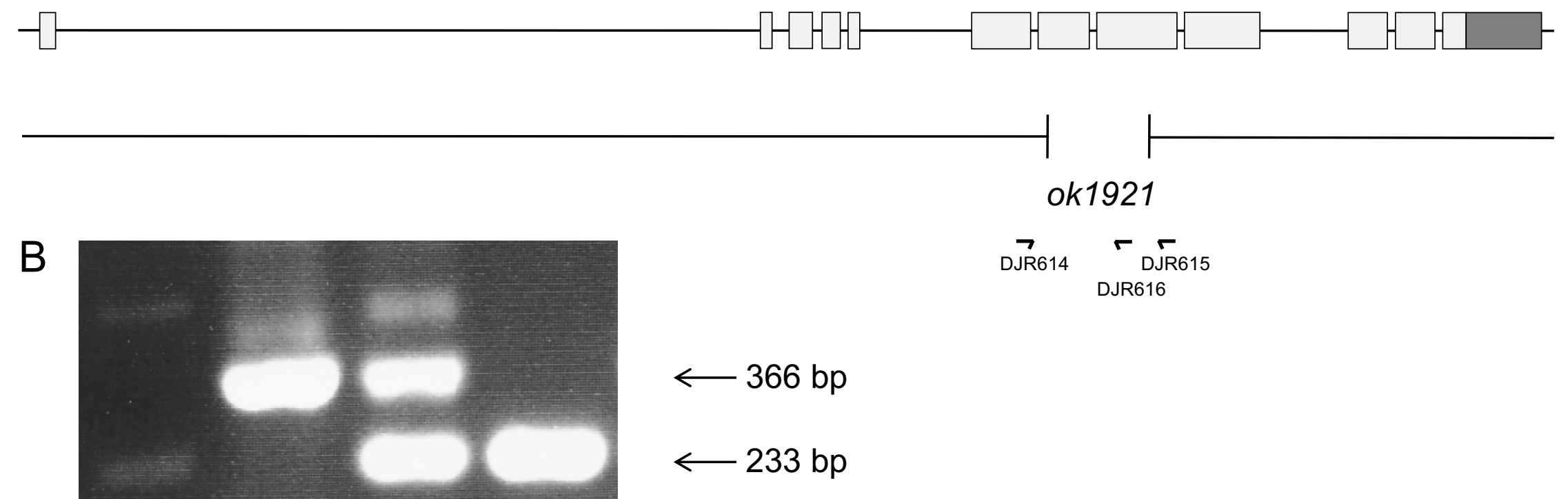

Figure S6: PCR detection of $\mathbf{r g l - 1}(\mathbf{o k 1 9 2 1})$. A) A scale schematic of the rgl-1 gene, the ok1921 lesion, and detection primers. B) Agarose gel of $+/+$, ok1921/+ and ok1921/ok1921 single animal PCR reactions. 
A
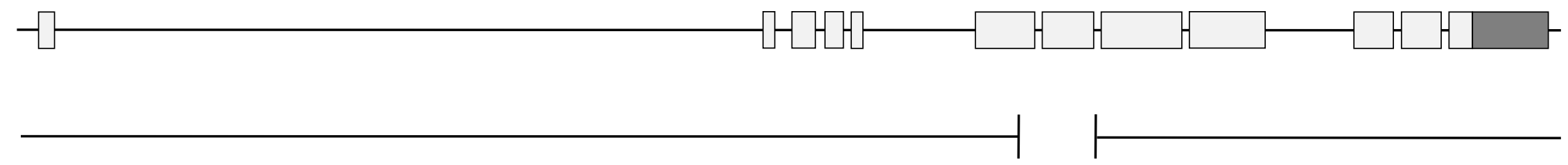

\section{B}

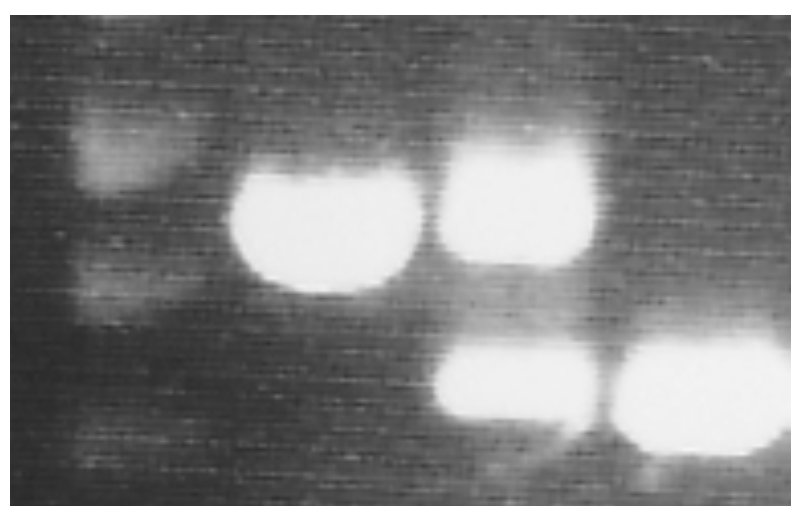

$\operatorname{tm} 2255$
$\underset{\text { TZ20 }}{\neg}$
DJR614

$\longleftarrow 913 \mathrm{bp}$

$\longleftarrow 595 \mathrm{bp}$

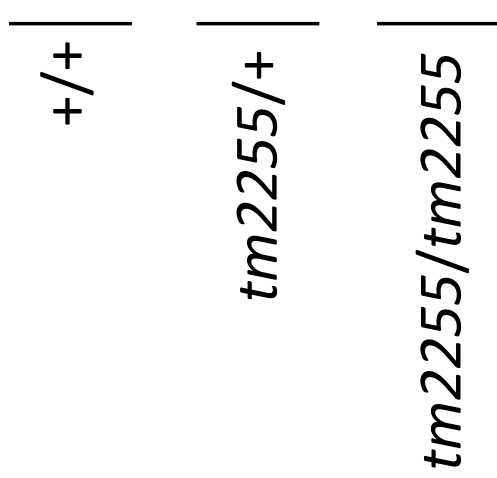

Figure S7: PCR detection of $\mathbf{r g l - 1 ( t m 2 2 5 5 ) . ~ A ) ~ A ~ s c a l e ~ s c h e m a t i c ~ o f ~ t h e ~} r g l-1$ gene, the $t m 2255$ lesion, and detection primers. B) Agarose gel of $+/+$, tm2255/+ and tm2255/tm2255 single animal PCR reactions. 